\title{
Genome-wide transcriptome profiling reveals functional networks involving the Plasmodium falciparum drug resistance transporters PfCRT and PfMDR1
}

Sophie H. Adjalley ${ }^{1,5}$, Daniel Scanfeld ${ }^{1,6}$, Elyse Kozlowski ${ }^{2,7}$, Manuel Lliná2 ${ }^{2,3}$ and David A. Fidock ${ }^{1,4^{*}}$

\begin{abstract}
Background: The acquisition of multidrug resistance by Plasmodium falciparum underscores the need to understand the underlying molecular mechanisms so as to counter their impact on malaria control. For the many antimalarials whose mode of action relates to inhibition of heme detoxification inside infected erythrocytes, the digestive vacuole transporters PfCRT and PFMDR1 constitute primary resistance determinants.

Results: Using gene expression microarrays over the course of the parasite intra-erythrocytic developmental cycle, we compared the transcriptomic profiles between P. falciparum strains displaying mutant or wild-type pfcrt or varying in pfcrt or pfmdr1 expression levels. To account for differences in the time of sampling, we developed a computational method termed Hypergeometric Analysis of Time Series, which combines Fast Fourier Transform with a modified Gene Set Enrichment Analysis. Our analysis revealed coordinated changes in genes involved in protein catabolism, translation initiation and DNA/RNA metabolism. We also observed differential expression of genes with a role in transport or coding for components of the digestive vacuole. Interestingly, a global comparison of all profiled transcriptomes uncovered a tight correlation between the transcript levels of pfcrt and pfmdri, extending to dozens of other genes, suggesting an intricate regulatory balance in order to maintain optimal physiological processes.

Conclusions: This study provides insight into the mechanisms by which $P$. falciparum adjusts to the acquisition of mutations or gene amplification in key transporter loci that mediate drug resistance. Our results implicate several biological pathways that may be differentially regulated to compensate for impaired transporter function and alterations in parasite vacuole physiology.
\end{abstract}

Keywords: Plasmodium falciparum, Antimalarial drug resistance, Comparative transcriptomics, Transporters, Fast fourier transform, Gene set enrichment analysis, Hypergeometric analysis of time series

\section{Background}

With an estimated 214 million clinical cases and 438,000 deaths in 2015 [1] malaria remains one of the deadliest infectious diseases. Clinical manifestations are due to successive rounds of Plasmodium parasite invasion, replication within host erythrocytes, egress and re-invasion. Present efforts to reduce mortality and morbidity focus

\footnotetext{
*Correspondence: df2260@columbia.edu

'Department of Microbiology and Immunology, Columbia University Medical Center, New York, NY 10032, USA

${ }^{4}$ Division of Infectious Diseases, Department of Medicine, Columbia

University Medical Center, New York, NY 10032, USA

Full list of author information is available at the end of the article
}

on the use of curative and preventive drug treatments as well as on vector control strategies [2]. Those include first-line artemisinin-based combination therapies (ACTs) that pair a highly potent artemisinin derivative with a longer-acting partner drug [3]. ACTs currently constitute the best regimens to treat 4aminoquinoline- and antifolate-resistant Plasmodium falciparum parasites that have swept across the world. While 20 years were necessary for chloroquine (CQ) resistance to spread from its origins in Southeast Asia to Africa [4], resistance to the antifolate combination of sulfadoxine-pyrimethamine disseminated at a much 
faster pace [5]. The recent emergence of delayed parasite clearance following artesunate or ACT administration in Western Cambodia and Thailand [6-8] reemphasizes the need to develop therapeutic strategies to treat drug-resistant $P$. falciparum malaria and define the molecular basis of resistance.

Two key genetic determinants of $P$. falciparum resistance to antimalarial compounds are pfcrt [9] and pfmdr1 $[10,11]$, which encode transporter proteins that localize to the digestive vacuole membrane of intra-erythrocytic parasites. While their native biological functions remain unknown, the inability to genetically disrupt $p f c r t$ or $p f m d r 1$ provides evidence for their essentiality $[12,13]$. Laboratory and field-based studies have demonstrated that PfCRT mutations constitute the primary determinant of in vitro CQ resistance and are associated with a substantially increased risk of CQ treatment failure in patients [14-16]. Studies with transgenic parasites or heterologous expression systems have attributed PfCRTmediated CQ resistance to drug efflux out of the low-pH digestive vacuole, where $\mathrm{CQ}$ becomes diprotonated, concentrates up to 1,000-fold and acts by binding to heme products and preventing heme detoxification [17-20]. PfCRT mutations can also mediate cross-resistance to amodiaquine and increase susceptibility to lumefantrine, mefloquine and artesunate [15, 21, 22]. pfmdr1 mutations as well as copy number variations have also been shown to impact $P$. falciparum susceptibility to several antimalarials [13, 22-26] presumably via drug transport across the digestive vacuole [27].

For PfCRT and PfMDR1, acquired resistance mutations, or in the case of $p f m d r 1$ gene amplifications, are thought to generally impart a fitness cost [28-31], presumably via altering their endogenous transport properties to the detriment of normal parasite development and replication. In endemic settings, this fitness cost manifests as a reduced prevalence of mutant pfcrt or pfmdr1 following the release of selective drug pressure [32-36]. However, additional mutations elsewhere in an organism can compensate for the cost of acquiring resistance $[37,38]$ and restore fitness, as shown for $P$. falciparum resistance to antifolates [39-41]. Furthermore, the long-term propagation of drug-resistant strains even in the absence of selective pressure suggests adaptive mechanisms that counteract the detrimental effects of resistance-conferring genetic changes [42].

Intriguingly, the question of how parasites transcriptionally adjust in the long-term to the acquisition of mutations or gene amplifications in drug resistance transporters has scarcely been addressed. An earlier comparison of the transcriptional profiles of pfcrt mutant strains generated by CQ selection from a drugsensitive parasite line suggested a limited number of changes in the parasite transcriptome [43]. That study, however, was restricted to a single time point of $P$. falciparum development within the host erythrocytes and as such it could not correct for temporal differences in the intra-erythrocytic developmental cycle (IDC) between parasite lines. Indeed, while transcriptome studies have suggested an inability of the parasite to transcriptionally respond to short-term drug pressure [44, 45], conceivably parasites may be able to evolve over multiple generations to states of optimal growth that adjust for physiological impacts of altered sequence or expression of drug/solute transporters. This is a testable hypothesis for PfCRT and PfMDR1, whose expression kinetics are closely tied to the formation of the parasite digestive vacuole during the trophozoite stage. Changes in the transcriptome profile of these mutant lines can be assessed in comparison with the carefully documented timeline of tightly regulated gene expression patterns that characterize the $P$. falciparum IDC [46-48]. These expression patterns underpin the biological processes of intracellular parasite development, replication and egress with accompanying morphological changes as parasites progress from rings to trophozoites to schizonts.

Here, we investigated how parasites adjusted transcriptionally to genetic modification of pfcrt or pfmdr1, resulting in altered drug resistance phenotypes following long-term culture. Using DNA microarrays, we conducted a genome-wide transcriptional profiling of genetically modified isogenic $P$. falciparum strains with either distinct PfCRT haplotypes or pfmdrl functional copy numbers throughout their 48-hour IDC. These previously engineered lines either display a loss of CQ resistance following the removal of the K76T mutation [20] (used epidemiologically as the primary molecular marker of $\mathrm{CQ}$ resistance) or show increased susceptibility to multiple antimalarial drugs (including mefloquine, lumefantrine, quinine and artemisinin) following genetic disruption of one of the two pfmdr1 copies present in the multidrug resistant FCB line [26]. To analyze the data we developed a computational method that combines Fast Fourier Transform (FFT) and Gene Set Enrichment Analysis (GSEA) to correct for temporal differences between samples and compare microarray data between time series. We also used a reference pool of 11 profiled parasite lines to distinguish between stochastically variant genes and gene expression differences of biological significance, including those of subtle amplitude. Our results revealed a striking and previously unreported correlation between the expression levels of pfcrt and $p f m d r 1$, and identified several pathways whose regulation was altered in response to changes in either gene, reinforcing the hypothesis of their functional interplay and the existence of coordinated functional networks. 


\section{Results}

A Fast Fourier Transform-based method to align microarray time series data between profiled isogenic parasite cell lines

To assess the genomic impact of $P$. falciparum acquiring genetic changes in transporters that mediate antimalarial drug resistance, we profiled the transcriptome of multiple isogenic lines characterized by altered pfcrt and pfmdr1 gene expression levels and/or corresponding protein haplotypes (Table 1). We also transcriptionally profiled the parental strains and performed both gene-specific and combined comparative analyses of the data sets.

The first data set (focusing on pfmdr1) included $\mathrm{FCB}^{p f m d r 1_{-} 3^{\prime} K D}$, which was engineered via single-site crossover-based disruption of one of the two pfmdr1 copies within the duplicated $100-\mathrm{kb}$ genomic region of the multidrug-resistant parasite strain FCB [26, 49]. This resulted in a $50 \%$ decrease in $p f m d r 1$ gene expression and a reduction of approximately $40 \%$ in its protein level (Additional file 1: Figure S1A). In addition to $\mathrm{FCB}^{\text {pfindr1_3'KD }}$ and its parental line FCB, our transcriptome analysis included 106/1, a parasite strain that genetically is nearly identical to FCB [50]. FCB and $\mathrm{FCB}^{\text {pfmdr1_3'KD }}$ display the same PfCRT haplotype (including the $\mathrm{K} 76 \mathrm{~T}$ mutation that is required for $\mathrm{CQ}$ resistance), whereas 106/1 has a key difference at position 76 that expresses a lysine $(\mathrm{K})$ and confers CQ susceptibility $[9,51]$.

The second data set $(p f c r t)$ included $7 \mathrm{G} 8^{p f c r t} T 76 K$ and $7 \mathrm{G} 8^{p f c r t+C T L}$ parasite lines that were generated by allelic exchange via single crossover recombination targeting codon 76 of the mutant pfcrt allele in the CQ-resistant strain 7G8 [20]. The encoded threonine residue was back mutated to the wild-type lysine in $7 \mathrm{G}^{\text {pfcrt_T76K }}$, resulting in a complete loss of CQ resistance, whereas it remained identical to $7 \mathrm{G} 8$ in the recombinant control $7 \mathrm{G} 8^{\text {pfcrt_CTL }}$. Additionally, the two recombinant lines are characterized by a two-fold reduction in pfcrt mRNA and protein expression, allowing us to assess the impact of both the acquisition/loss of a point mutation and alteration in gene expression (Additional file 1: Figure $\mathrm{S} 1 \mathrm{~B})$. Of note, $7 \mathrm{Gr}^{\text {pfcrt_CTL }}$ is only moderately CQresistant, with a mean $\mathrm{CQ} \mathrm{IC}_{50}$ value $(109 \mathrm{nM})$ that is close to half that of the parental $7 \mathrm{G} 8$ line (251 nM) by virtue of the reduced PfCRT expression [20].

To conduct our comparative analysis, tightly synchronous parasites were cultured for the length of a complete IDC ( $\sim 8 \mathrm{~h})$. RNA was extracted from samples collected every $6 \mathrm{~h}$ for $48 \mathrm{~h}$ and assayed on a 70-mer DNA microarray, representing more than 5,350 P. falciparum genes [52]. Alignment of each profiled time series to the transcriptome of the reference strain 3D7 showed small but significant differences in the IDC duration. Indeed, the transgenic strains displayed a shorter life cycle than their respective parents (Fig. 1a).

Given the resulting slight time shifts between the time series, we developed a computational method to align the microarray expression data and to ensure that our comparative analysis would not be skewed by temporal differences in the developmental stage of the different parasite lines at the time of harvesting. We used FFT to impute gene expression levels at 376 unassayed time points to obtain a 7.5-minute resolution of each time course (384 time points in total; see Methods, Additional file 2: Table S1). To validate this approach, we first performed a simulation using published transcriptome data sets that constituted of hourly time course data for the

Table 1 Summary of the $P$. falciparum lines used in the study. Drug resistance phenotype and pfcrt/pfmdr1 phenotypes are indicated

\begin{tabular}{|c|c|c|c|c|c|c|c|c|c|c|c|c|c|c|c|c|c|c|c|}
\hline \multirow[b]{2}{*}{ Parasite Line } & \multirow[b]{2}{*}{ Parent } & \multirow[b]{2}{*}{ Rec. } & \multirow[b]{2}{*}{ Drug Phenotype } & \multirow[b]{2}{*}{ Genetic characteristic } & \multicolumn{9}{|c|}{ PfCRT haplotype } & \multicolumn{5}{|c|}{ PfMDR1 haplotype ${ }^{e}$} & \multirow[t]{2}{*}{$\overline{C N}$} \\
\hline & & & & & 72 & 74 & 75 & 76 & 220 & 271 & 326 & 356 & 371 & 86 & 184 & 1034 & 1042 & 1246 & \\
\hline FCB & - & No & $\begin{array}{l}\text { Multi-drug } \\
\text { resistant }^{a}\end{array}$ & 2 pfmdr 1 copies & $C$ & I & $\mathbf{E}$ & $\mathrm{T}$ & $\mathrm{S}$ & $\mathbf{E}$ & $\mathrm{S}$ & I & I & $N$ & Y & S & $\mathrm{N}$ & $\mathrm{D}$ & 2 \\
\hline $\mathrm{FCB}^{\text {pfmdrl }} 3^{3} K D$ & FCB & Yes & CQ-resistant $^{b}$ & 1 pfmdr 1 copy & $C$ & I & $\mathbf{E}$ & $\mathbf{T}$ & $\mathrm{S}$ & $\mathbf{E}$ & $\mathrm{S}$ & I & I & $\mathrm{N}$ & Y & $\mathrm{S}$ & $N$ & D & 1 \\
\hline $106 / 1$ & - & No & CQ-sensitive & $\begin{array}{l}2 \text { pfmdr1 copies, } \\
\text { pfcrt K76 }\end{array}$ & $\mathrm{C}$ & I & $\mathbf{E}$ & $\underline{K}$ & $\mathrm{~S}$ & $\mathbf{E}$ & $\mathrm{S}$ & । & I & $\mathrm{N}$ & Y & S & $\mathrm{N}$ & D & $2^{f}$ \\
\hline $7 \mathrm{G} 8$ & - & No & CQ-resistant & pfert K76T & $\mathrm{S}$ & $M$ & $\mathrm{~N}$ & $\mathrm{~T}$ & $\mathrm{~S}$ & $\mathbf{Q}$ & D & $\mathrm{L}$ & $\mathrm{R}$ & $\mathrm{N}$ & $\mathbf{F}$ & $C$ & D & $Y$ & 1 \\
\hline $7 G 8^{p f c r t \_C T L}$ & $7 \mathrm{G} 8$ & Yes & Less CQ-resistant $^{C}$ & $\begin{array}{l}\text { pfcrt shorter 5'UTR, } \\
\text { T76 }\end{array}$ & $\mathrm{S}$ & $M$ & $\mathrm{~N}$ & $\mathrm{~T}$ & $\mathrm{~S}$ & $\mathbf{Q}$ & D & $\mathrm{L}$ & $\mathrm{R}$ & $\mathrm{N}$ & $\mathbf{F}$ & $\mathrm{C}$ & D & $\mathrm{Y}$ & 1 \\
\hline $7 G 8^{p f c r t \_T 76 K}$ & $7 \mathrm{G} 8$ & Yes & CQ-sensitive $^{d}$ & $\begin{array}{l}\text { pfcrt shorter 5'UTR, } \\
\text { K76 }\end{array}$ & $\mathrm{S}$ & $M$ & $\mathrm{~N}$ & $\underline{K}$ & $\mathrm{~S}$ & $\mathbf{Q}$ & D & $L$ & $\mathrm{R}$ & $\mathrm{N}$ & $\mathbf{F}$ & $C$ & D & $\mathbf{Y}$ & 1 \\
\hline
\end{tabular}

CQ: chloroquine,CN: pfmdr1 copy number, CTL: control, KD: knockdown, Rec: Recombinant, UTR: Untranslated region

${ }^{a}$ Resistant to chloroquine through pfcrt mutations and partially resistant to lumefantrine, mefloquine and quinine through pfmdr1 gene amplification

${ }^{b}$ Genetic ablation of 2 nd pfmdr1 copy number led to increased susceptibility to lumefantrine, mefloquine and quinine

'Reduced pfcrt expression through replacement of full-length 5'UTR with shorter version resulted in reduced level of CQ resistance

${ }^{\mathrm{d}}$ Reversion of PfCRT K76T to wild-type K76 led to loss of CQ resistance

eAmino acids that differ from wild-type haplotypes (reference sequence 3D7, not shown) are shown in bold

${ }^{\mathrm{f}}$ Transcriptome and qRT-PCR studies indicated that 106/1 expresses the equivalent of a single pfmdr1 copy, despite having two physical copies 

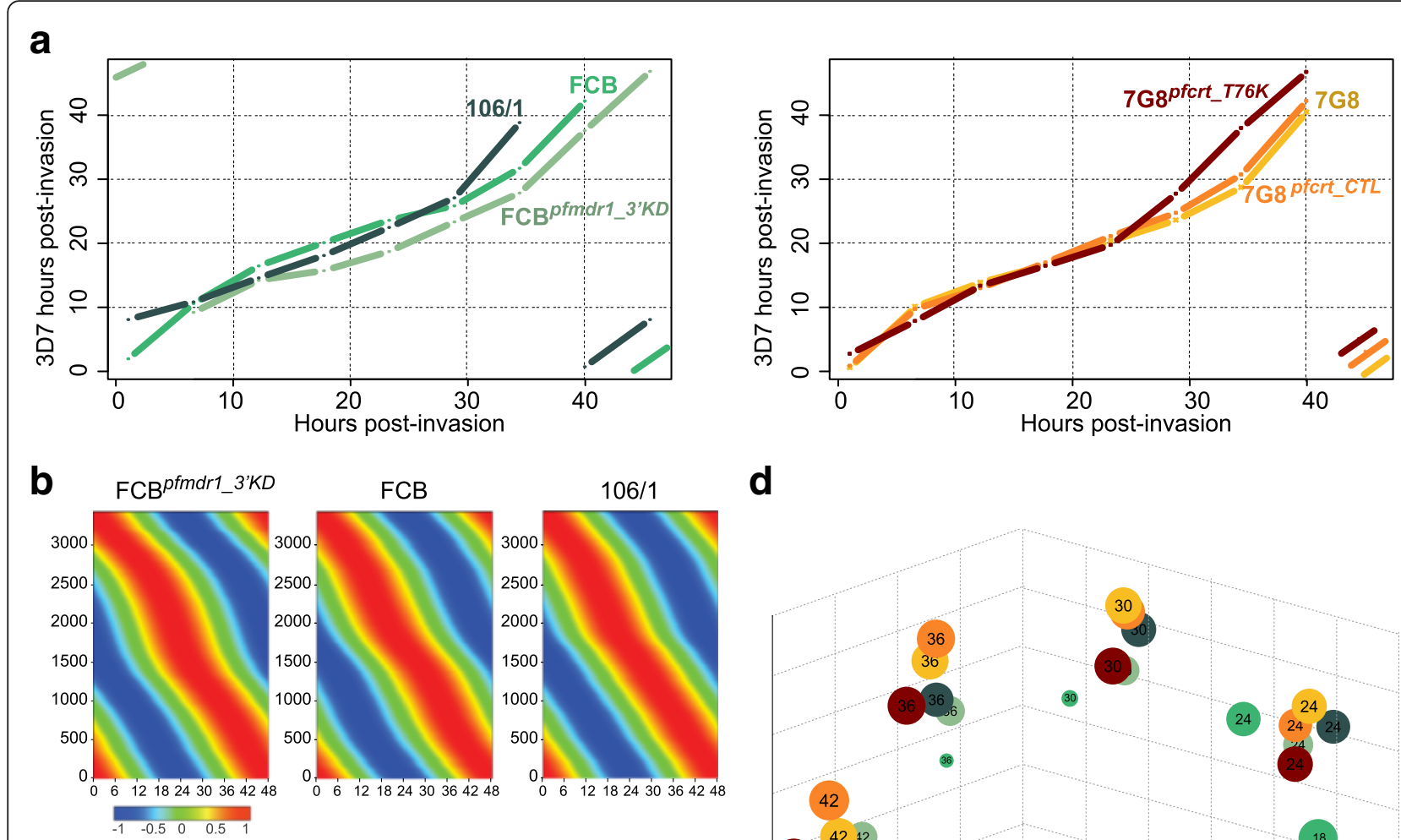

d
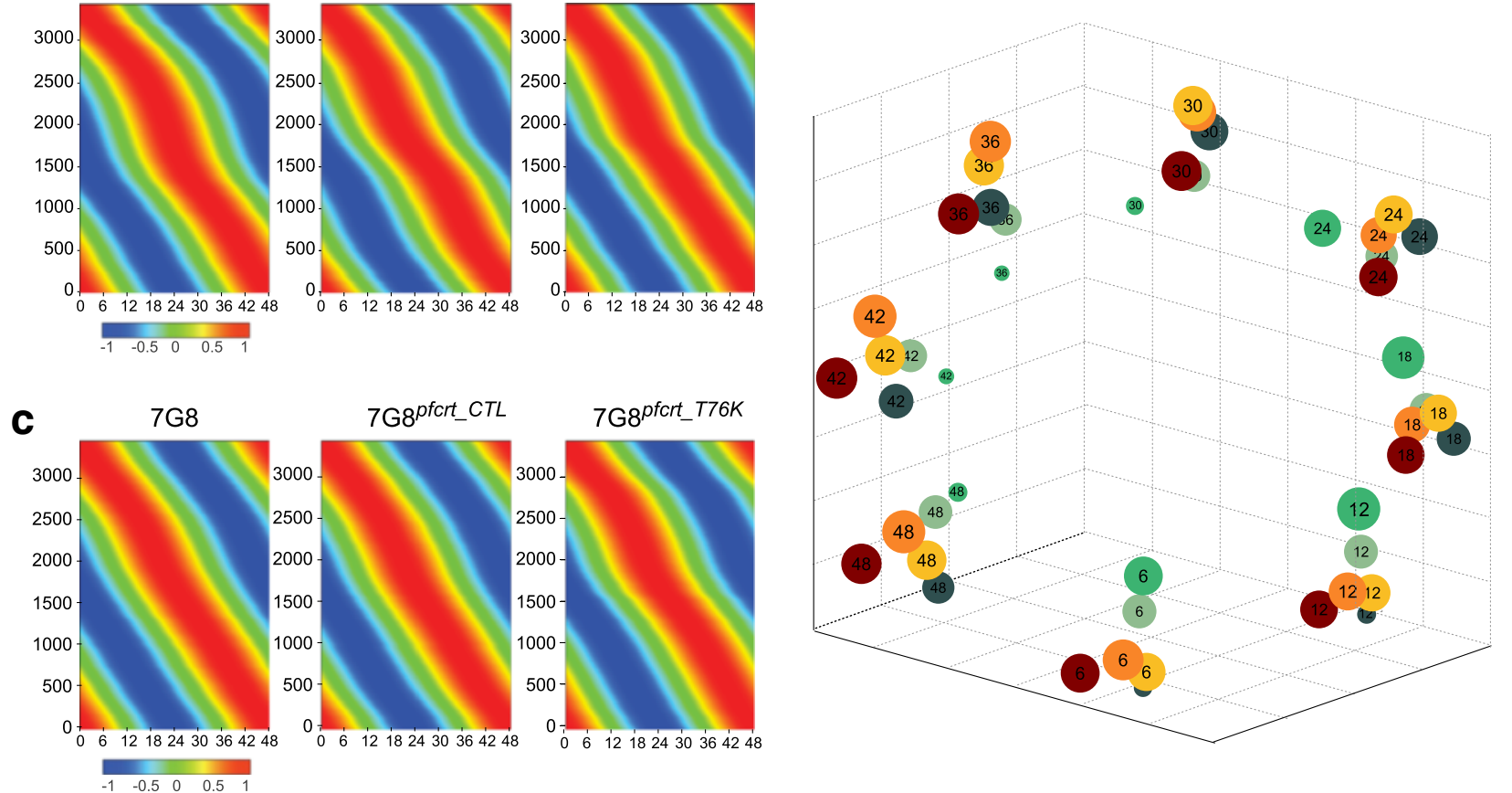

Fig. 1 Alignment of all time series to the 3D7 reference transcriptome reveal differences in growth rates between the profiled parasite lines. a Plot of all time courses before temporal alignment of the transcriptome data. For each transcriptome data set, the original 8 time points of each 48-hour time course (corresponding to a single intra-erythrocytic developmental cycle (IDC)) were aligned to the transcriptome of 3D7 that earlier had been elucidated at a one-hour resolution [46]. b Phaseograms of all parasite lines in the FCB set after dynamic time warping. Genes (on the Y-axis) were ordered according to their phase of expression computed using Fast Fourier Transform (FFT), which interpolated expression levels at unassayed time points. The resulting output generated a smooth sine curve with a single peak of maximum expression (shown in red, spanning a heatpmap gradient to the minimum transcript levels shown in blue) for each gene across the entire 48-hour IDC. c Phaseograms of the three parasite lines in the 7G8 set after dynamic time warping. d Principal Component Analysis (PCA) confirming the proper alignment of all transcriptomes. The $\sim 3,000$ to $\sim 3,500$ genes represented in each transcriptome were used to generate a matrix of values that was reduced to a tri-dimensional projection using PCA. The first two principal components accurately recover the progression of the lines through the 48-hour lifecycle while the third principal component focuses on differences between the lines as symbolized by the size of the circles. Circle colors refer to the parasite lines depicted in (a)

strains HB3, Dd2 and 3D7 [48]. For each strain we selected 8 time points spread $6 \mathrm{~h}$ apart, from which we generated a curve of 376 imputed points. Using Pearson correlation values for gene expression, we then compared each actual hourly time point to the imputed curve to identify the closest matching time point. We observed that actual time points from the original time courses matched imputed time points that were fairly evenly spread along the imputation-derived curve, with correlations of average 83$91 \%$ across the range of time points for each strain. This suggests that the imputation method can work on datasets that comprise only eight time points, such as ours.

Using our imputed data sets, we then applied a dynamic time warping/Pearson Correlation Coefficient 
(PCC) method to determine for each profiled parasite line the optimal post-invasion time point (corresponding to the first sampling time point) along the IDC (see Methods). All time courses were then realigned to the 3D7 transcriptome and to each other when performing pairwise comparisons (Additional file 3: Figure S2).

Phaseograms representing the transcriptome of each strain were assembled by hierarchically clustering the mean-centered $\log _{2}$ expression ratios of the genes that passed the different filters ( $\sim 3,500$ genes), according to their phase of expression calculated as described above. Transcriptomes of all strains reproduced the cascade of expression typical of $P$. falciparum, in which genes are expressed in a highly periodic mode [46] (Fig. 1b, c). Furthermore, all profiled strains demonstrated excellent synchronicity, with tight clustering of the expression data as observed using Principal Component Analysis (PCA), thereby validating the alignment of the time series (Fig. 1d).

\section{Chromosomal mapping of gene expression changes reveals amplicon loss on chromosome 5 in $\mathrm{FCB}^{\text {pfmdr1_3' } K D}$ and down-regulation of a chromosome 2 gene cluster in $7 \mathrm{G}^{\text {pfcrt_T76K }}$}

To identify genes that were differentially expressed between parasite time series within each data set, steady state transcript levels were analyzed across the IDC for each profiled strain and compared to each other. We computed gene expression fold differences for each pairwise comparison as the difference between the areas under the curves, which were extracted from absolute temporal gene expression plots derived from the full 384 time point data.

We then generated chromosome maps in which fold changes in expression were plotted as a function of the chromosomal position of each gene. These maps revealed clusters of differential expression in telomeric regions of several chromosomes, possibly indicating hypervariable transcription of genes within these regions, as previously reported [53] (Additional file 4: Figure S3). Surprisingly, an entire set of genes all located on chromosome 5 and overlapping with the 100-kb amplicon containing pfmdr1 (PF3D7_0523000) appeared to be differentially expressed between FCB and both $\mathrm{FCB}^{\text {pfmdri_3 }}{ }^{\prime} K D$ and $106 / 1$ (Fig. 2a, b). All genes within this region except PF3D7_0521300, PF3D7_0521800, PF3D7_0522900, and PF3D7_0523100 were expressed two- to four-fold less in $\mathrm{FCB}^{\text {pfmadr }{ }_{-} 3^{\prime} K D}$ and 106/1 as compared to FCB (Fig. 2a, b; Additional file 5: Table S2). Interestingly, global gene expression profiles within the amplicon region on chromosome 5 were

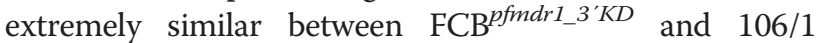
(Fig. 2a, b). Given the overlap with genes that constitute the amplicon in the FCB strain (Additional file 6: Figure
S4), we performed quantitative real time PCR on parasite genomic DNA to assess whether the observed transcriptional differences between $\mathrm{FCB}$ and both $\mathrm{FCB}^{\text {ffmdr }}{ }^{3}{ }^{\prime} K D$ and 106/1 originated from different copy numbers within the pfmdr1 region on chromosome 5. Quantitative PCR results indicated that the $\mathrm{FCB}^{p f m d r{ }_{1}{ }^{\prime} K D}$ strain has a single copy of the genes that are found duplicated in FCB, including pfmdr1. Furthermore, although our microarray data showed a similar down-regulation of these genes in 106/1, the parasite strain harbored two physical copies of these. These results with 106/1 indicate a different mechanism for reduced gene expression in that chromosomal region, potentially involving silencing of one of the two amplicons (Additional file 7: Table S3).

The same chromosomal mapping of gene expression fold changes revealed the down-regulation of a cluster of genes on chromosome 2 in the back-mutant strain $7 \mathrm{G}^{\text {pfcrt_T76K }}$ in comparison to the background strain 7G8 (Fig. 2c; Additional file 8: Table S4). This downregulation was specific to the back-mutant parasites, as no gene expression difference was observed in that region when comparing the transgenic control strain 7 G8 $8^{\text {pfcrt_CTL }}$ to $7 \mathrm{G8}$ (Fig. 2d). Interestingly, many of the affected genes encode proteins involved in parasite invasion of host erythrocytes, including Merozoite Surface Proteins (PF3D7_0206800 and PF3D7_0207000) and Serine Repeat Antigen (SERA) gene products (PF3D7_0207400, PF3D7_0207500, PF3D7_0207600, PF3D7_0207700, and PF3D7_0207800) (Fig. 2c, Additional file 8: Table S4).

\section{Genetic or transcriptional deamplification of the pfmdr1- containing amplicon has broad transcriptional consequences}

To assess the transcriptional variations between the profiled strains, we performed gene expression and gene set enrichment analyses, considering both absolute and normalized gene expression fold changes (Methods). We also conducted pairwise comparisons of the transcriptome data sets for three developmental stage windows, corresponding to rings (12 to $16 \mathrm{~h}$ post-invasion), trophozoites ( 24 to $28 \mathrm{~h}$ post-invasion) and schizonts (32 to $36 \mathrm{~h}$ post-invasion. These comparisons enabled us to detect changes in genes/gene sets within a short window of expression or genes/gene sets whose peak of expression occurred at different times between related parasite lines (Methods; Additional file 9: Figure S5). We observed that more than $70 \%$ of all genes peaked within the same 8-hour window in the pfmdr1 dataset while about $80 \%$ of the genes peaked within the same 5-hour window in the pfcrt dataset (Additional file 10: Table S5). Overall, the great majority of the observed changes was driven by changes in gene expression levels as opposed to altered timing of expression. 


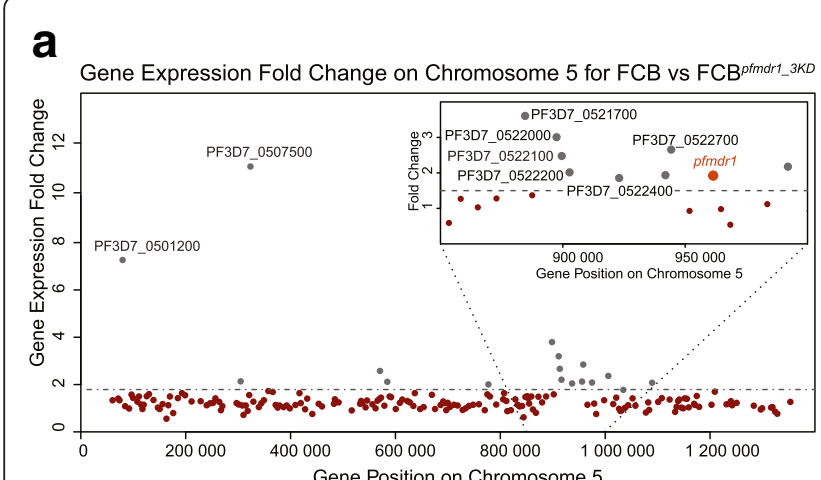

b

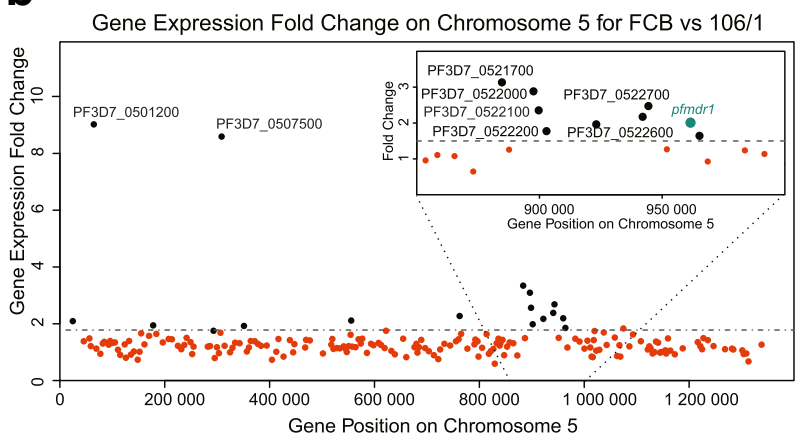

C

d

Gene Expression Fold Change on Chromosome 2 for $7 \mathrm{G} 8$ vs $7 \mathrm{G}^{\text {pfert_t76K }}$
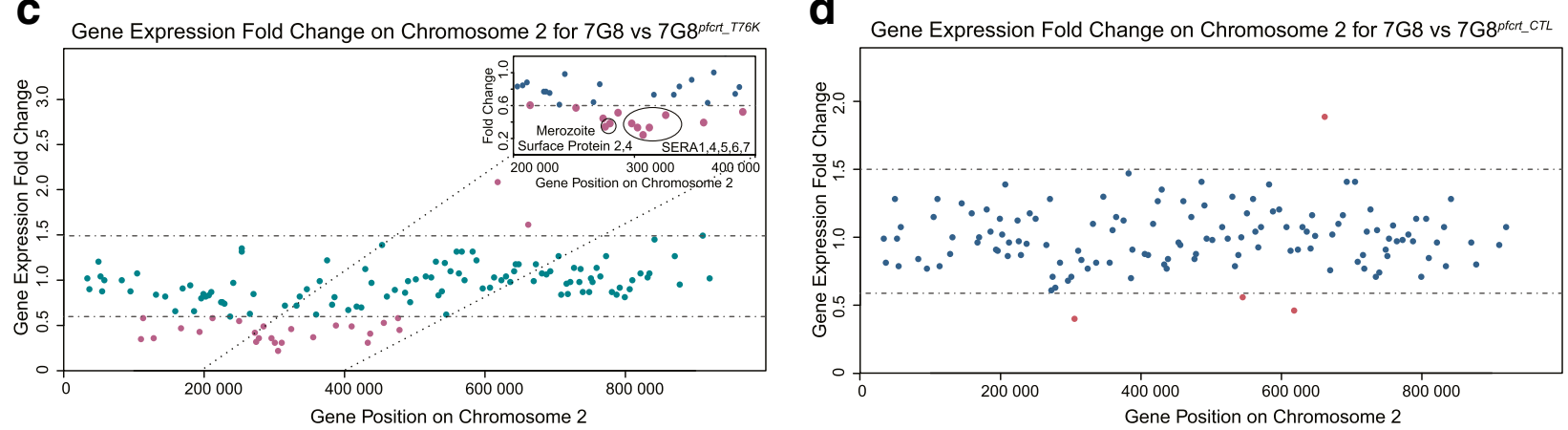

Fig. 2 Differential gene expression detected by chromosome mapping. a Chromosome mapping of gene expression fold differences between FCB and FCB ${ }^{\text {ffmdrl } 3_{3}{ }^{\prime} K D}$ shows differential transcription of a large fragment on chromosome 5 corresponding to the pfmdr 1 -containing 100 -kb amplicon region. For each gene located on chromosome 5, the expression fold difference between FCB and FCB $B^{\text {pfmdr } 13^{3} K D}$ was mapped using the chromosomal coordinates. Interestingly, PF3D7_0507500 (a subtilisin-like protease) and PF3D7_0501200 (a parasite-infected erythrocyte surface protein) show higher expression in FCB in comparison to both FCB ${ }^{\text {pfmdr1_ } 3^{\prime} K D}$ and 106/1 (see panel B). The inset shows the genes within the pfmdr1-containing amplicon region. $\mathbf{b}$ Chromosome mapping of gene expression fold differences between FCB and 106/1 for each gene on chromosome 5 with the inset showing genes within the pfmdr1-containing amplicon region. c Chromosome mapping of gene expression fold differences between $7 \mathrm{G} 8$ and $7 \mathrm{G} 8^{\text {pfcrt_T76K }}$ shows differential gene expression of a large number of genes located on chromosome 2 (inset shows a cluster of genes coding for proteins involved in parasite invasion). $\mathbf{d}$ Chromosome mapping of gene expression fold differences between $7 \mathrm{G} 8$ and $7 \mathrm{G} 8^{\text {pfrt_CTL }}$ for each gene on chromosome 2. In each pairwise comparison, the fold difference in gene expression was calculated as the difference between the areas under the curve computed on the full 384 time point data

Analysis of the $p f m d r 1$ data set (FCB vs. $\mathrm{FCB}^{\text {pfmdr }{ }_{-} 3^{\prime} K D}$ and FCB vs. 106/1) showed that many of the genes with significant differences in expression level encoded proteins of unknown function. This analysis also revealed decreased expression of multiple genes involved in RNA and protein metabolism upon deamplification of the pfmdr1containing amplicon region on chromosome 5 (Fig. 3a, b). Among these were genes coding for RNA-binding proteins, splicing/exosome elements and protein chaperones and modifiers (Additional file 11: Table S6A,B). In contrast, numerous genes encoding ribosomal protein subunits, or involved in RNA translation initiation or protein turnover showed a more than two-fold higher expression level in both strains with lower pfmdr1 expression (Fig. 3c, d). Furthermore, genes mainly involved in transcriptional control, including several elements of the basal transcription machinery, were 2 to 3.5-fold up-regulated in $\mathrm{FCB}^{\text {pfindr1_ }}$ 3'KD $^{\text {'K }}$ and $106 / 1$ in comparison to FCB. For instance, those encoding a putative RNA polymerase II
(PF3D7_1027400), and the AP2-containing transcription factors PF3D7_0730300 and PF3D7_0613800 [54, 55] displayed higher expression in $\mathrm{FCB}^{\text {pfm }} \overline{\mathrm{dr}} 1_{-} 3^{\prime} \mathrm{KD}$ (Additional file 11: Tables S6C). Genes encoding chromatin constituents such as the components of the nucleosome core histone H3 (PF3D7_0610400) and the histone variant H2B (PF3D7_0714000) were 2 to 2.5-fold less expressed in FCB in comparison to 106/1 (Additional file 11: Table S6D). These results suggest an alteration of the global transcriptional and translational activities in both strains with reduced pfmdr1 expression in comparison to FCB.

Multiple genes implicated in cellular metabolism, parasite remodeling and invasion also displayed higher transcript levels in $\mathrm{FCB}^{p f m d r{ }_{1} 3^{\prime} K D}$ and 106/1 compared to FCB (Fig. 3c, d, Additional file 11: Table S6C,D). These observations were confirmed by applying a statistical approach that we termed Hypergeometric Analysis of Time Series (HATS), which combined Fast Fourier Transform and GSEA, to further assess the functional 


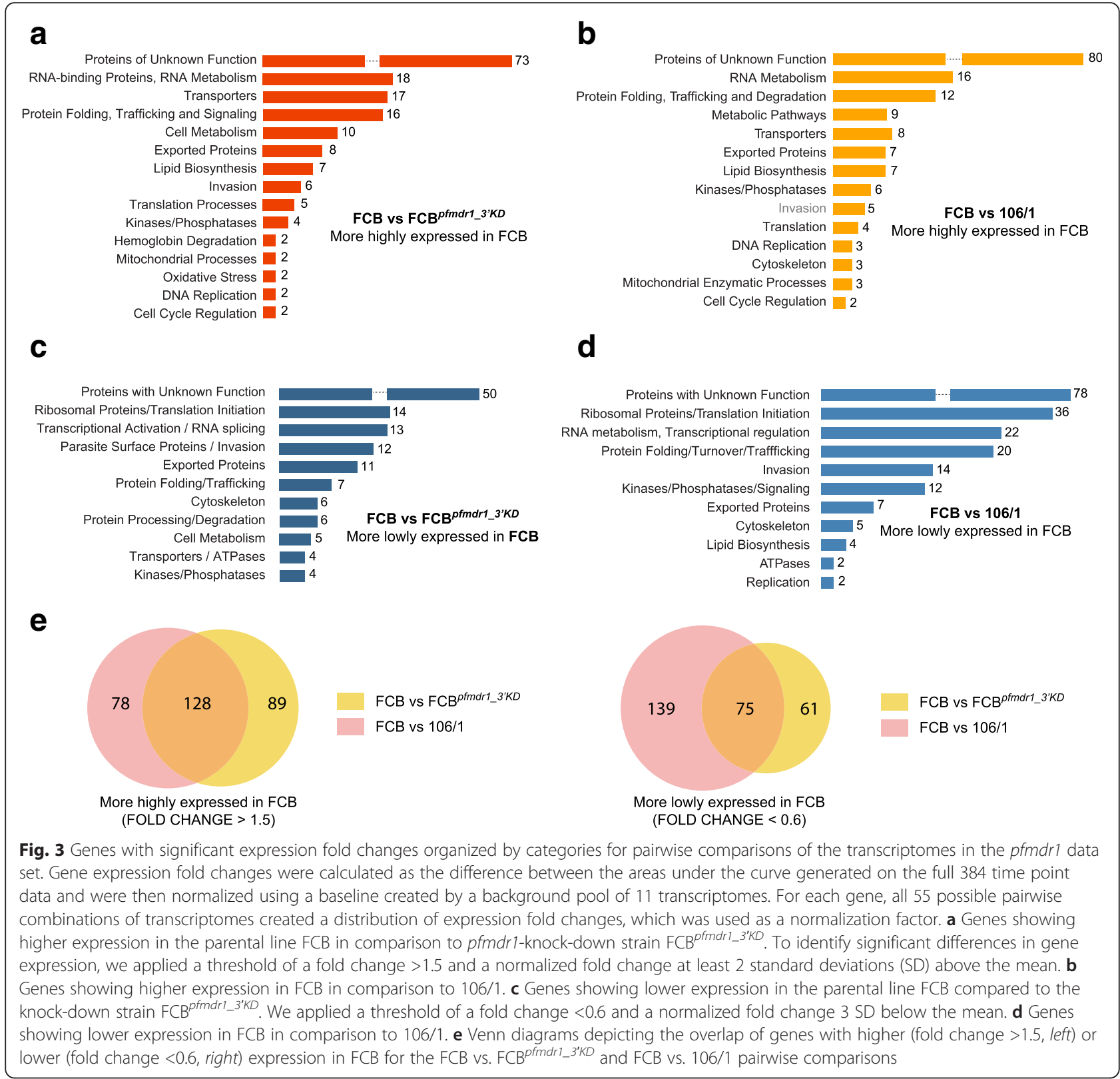

significance of the variations in gene expression observed between the parasite strains (see Methods). HATS, although based on GSEA, imputes a gene set enrichment score from the hypergeometric distribution of all gene sets calculated following 1,000 random permutations, instead of using a weighted Kolmogorov-Smirnov-like test [56]. HATS demonstrated that genes more highly expressed in

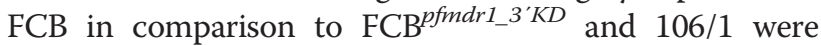
enriched mainly for metabolic and enzymatic activities such as purine/pyrimidine and pyruvate/glutamate metabolic pathways (Fig. 4a, b). In contrast, several genes and gene sets associated with transcription regulation, translation initiation including ribosome biogenesis, and proteasome-mediated protein catabolism/ turnover, were depleted in FCB in comparison to both strains with lower $p f m d r 1$ expression (Fig. 4c, d). Stageenrichment analysis (Methods) revealed that these differences were detectable mainly during the trophozoite and schizont stages (Fig. 4e, f, Additional files 12 and 13: Tables S7 and S8 respectively). Some of these alterations, including those affecting protein synthesis and protein turnover via proteasome degradation have also been observed in the transcriptional profiles of artemisinin-resistant patient isolates carrying causal mutations in the kelch gene K13, suggesting that these pathways might contribute to general mechanisms adopted by multi-drug resistant parasites [57].

Further stage-specific analysis showed that gene categories comprising transporters or components localized 


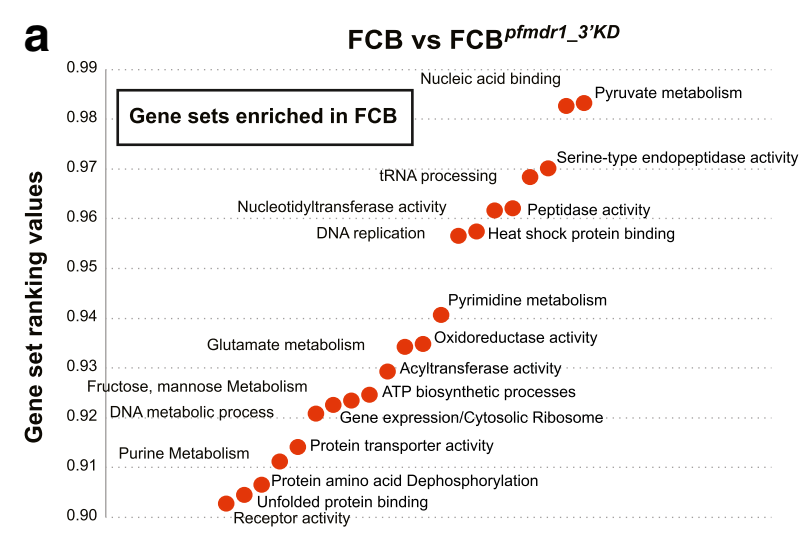

C FCB vs FCB ${ }^{\text {pfmdr1_3KD }}$

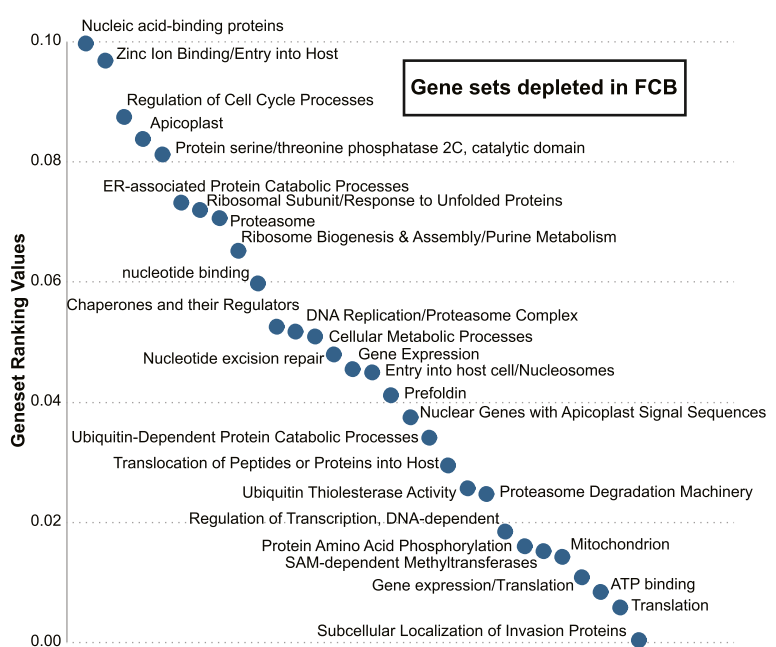

e

FCB vs FCB ${ }^{\text {pfmdr1 }} 33 K D$

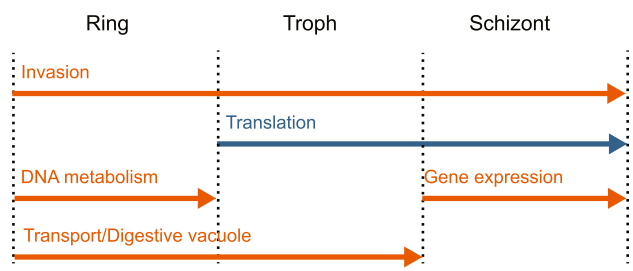

f

FCB vs $106 / 1$

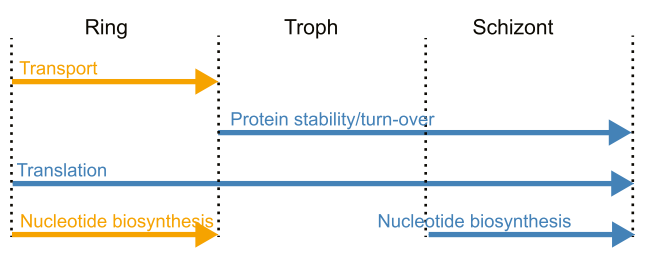

b

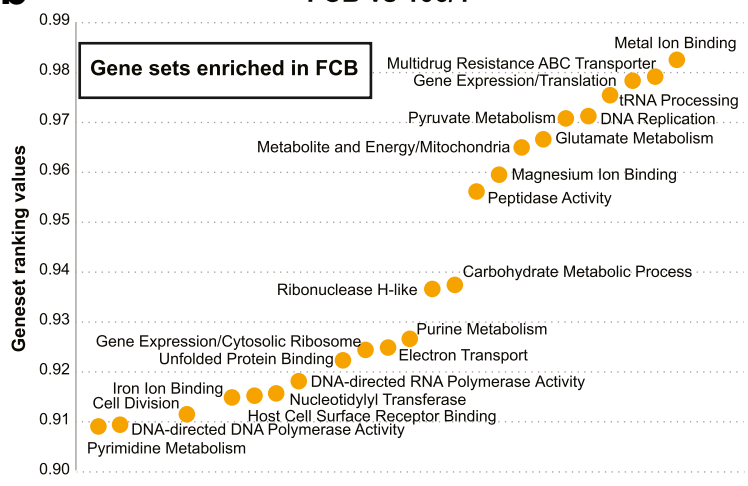

d

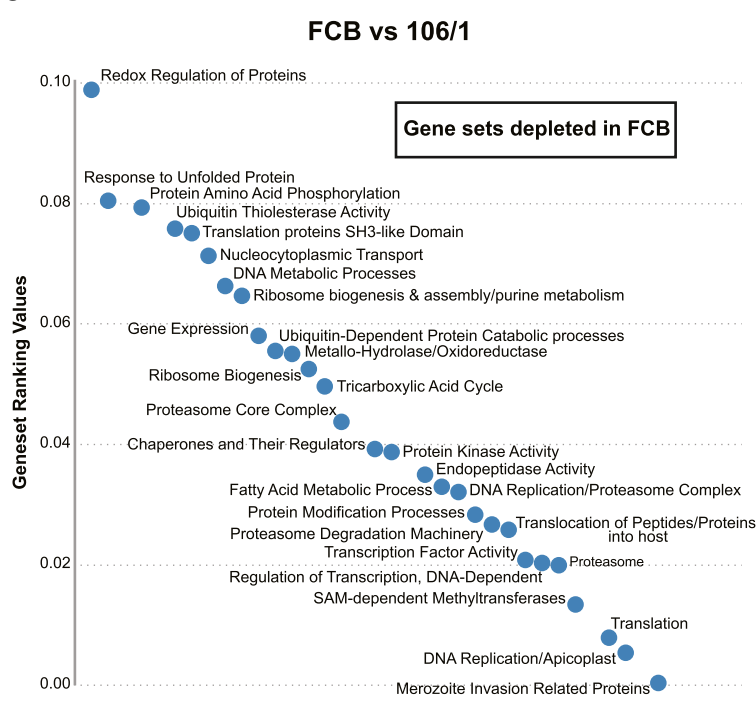

g

Enriched Gene sets (Mean ranking > 0.9)

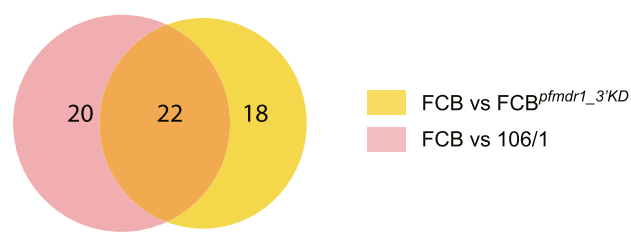

h

Depleted Gene sets (Mean ranking < 0.1)

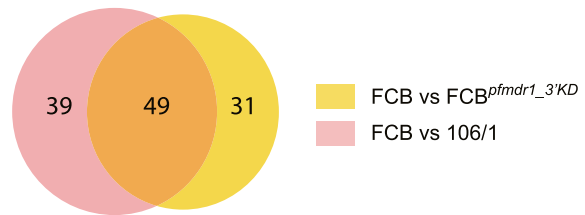

Fig. 4 (See legend on next page.) 


\section{(See figure on previous page.)}

Fig. 4 Hypergeometric Analysis of Time Series (HATS) for pairwise comparisons of the transcriptomes in the pfmdr 1 data set. HATS was performed by combining FFT to align the time series with a hypergeometric distribution approach based on random permutations to compute an enrichment score for each gene set. Normalization of the enrichment scores was performed using an expression baseline constituted by a background pool of 11 transcriptome data sets (see Methods). We applied a threshold of normalized gene set rank $>0.9$ for gene sets we considered positively enriched and normalized gene set rank <0.1 for gene sets considered negatively enriched. Gene sets were built using GO, KEGG and the Malaria Parasite Metabolic Pathway (listed in Additional file 26: Table S17). a Gene sets that are enriched in FCB in comparison to FCB ${ }^{\text {pfmdr1 } 3^{\prime} \text { KD }}$ (mean ranking values $>0.9$ ). b Gene sets that are enriched in FCB in comparison to 106/1 (mean ranking values $>0.9$ ). c Gene sets that are depleted in FCB in comparison to FCB ${ }^{\text {ffmdr } 13^{\prime} K D}$ (mean ranking values $<0.1$ ). d Gene sets that are depleted in FCB in comparison to 106/1 (mean ranking values $<0.1$ ). e Stage-associated enrichment analysis in FCB in comparison to FCB ${ }^{\text {pfmdr } 13^{\prime} K D}$. Gene sets with significant enrichment (orange) or depletion (blue) for three windows of the parasite IDC correspond to rings ( $\mathrm{R}, 12-16 \mathrm{~h}$ post-invasion), trophozoites (T, 24-28 h post-invasion) and schizonts (S, 32-36 h post-invasion). f Stage-associated enrichment analysis in FCB in comparison to 106/1. Gene sets with significant enrichment (orange) or depletion (blue) during the same three stages as in (E). $\mathbf{g}$ Venn diagrams depicting overlaps of gene sets significantly enriched in FCB (mean rank of enrichment score $>0.9$ ) for both FCB vs. FCB ${ }^{\text {pfmdr } 133^{\prime} K D}$ and FCB vs. 106/1 pairwise comparisons, as identified from our HATS analysis. $\mathbf{h}$ Venn diagrams depicting overlaps of gene sets significantly depleted in FCB (mean rank of enrichment score $<0.1$ ) for both FCB vs. FCB pfmdr $13^{3} K D$ and FCB vs. 106/1 pairwise comparisons, as identified using HATS

to the parasite digestive vacuole were significantly enriched (>2-fold) in FCB in comparison to $\mathrm{FCB}^{\text {pfmdr1_3 }}$. $K D$ in mature asexual blood stages, i.e. when the digestive vacuole is functioning. These genes included two V-type ATPases (PF3D7_1354400, PF3D7_0519200), an AAA family ATPase (PF3D7_0707400), an ABC transporter (PF3D7_0810200), and drug/metabolite and amino acid transporters (PF3D7_1428200 and PF3D7_1132500), (Fig. 4e, Additional file 12: Table S7A,C,G). These genes sometimes also displayed a higher expression in FCB in comparison to 106/1 (Fig. 4f, Additional file 13: Table S8A).

Overall, the global transcriptional response to the deamplification of the pfmdrl-containing amplicon was highly similar between $\mathrm{FCB}^{\text {pfmdr }{ }_{-3}{ }^{\prime} K D}$ and 106/1. For instance, expression analysis of individual genes revealed that both $\mathrm{FCB}^{\text {pfmdri_ }}{ }^{\prime} K D$ and $106 / 1$ strains in comparison to FCB showed a 2- to 3-fold downregulation of the genes coding for an ATP-dependent RNA helicase (PF3D7_0521700) and the H2 ribonuclease (PF3D7_0623900) (Additional file 11: Table S6E). These two genes were among the very few that were differentially expressed between the strains across all time points throughout the IDC, as demonstrated by stage-specific analysis (Additional files 12 and 13: Tables S7K and S8K respectively). HATS also identified several gene sets that were enriched or depleted in both $\mathrm{FCB}^{\text {pfmdri_3'KD }}$ and $106 / 1$ in comparison to $\mathrm{FCB}$, which included parasite transporters, components of the proteasome degradation machinery and ribosome constituents (Additional file 11: Table S6G,H). Overall, we observed an overlap of greater than $50 \%$ in the gene sets enriched or depleted in

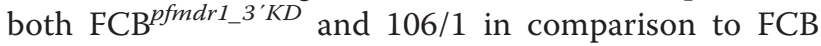
(Fig. 4g, h, Additional file 11: Table S6E,F). Interestingly, we also detected enrichment for gene sets associated with mitochondrial activities such as energy production and electron transport in the FCB versus $106 / 1$ pairwise comparison, but not when comparing
FCB with $\mathrm{FCB}^{\text {pfmdr1_3'KD }}$ (Fig. 4a, b). These gene sets in addition to those related to aminoacyl-tRNA biosynthesis, redox regulation or associated with the parasite digestive vacuole were differentially enriched between both $p f m d r 1$ knock-down strains (Additional file 14: Figure S6). The contrasting nature of the PfCRT haplotypes between the two strains might account for these differences. This aspect was further addressed when analyzing the $p f c r t$ data set.

\section{Drug-resistance mutations in pfcrt affect protein synthesis gene expression}

We applied analytical approaches similar to those described above to the pfcrt data set (Additional file 15: Table S9) and performed pairwise comparisons of 7G8, $7 \mathrm{G} 8^{\text {pfcrt_CTL }}$ and $7 \mathrm{G} 8^{\text {pfcrt_T76K }}$ transcriptomes. Expression analysis for individual genes showed that several genes encoding proteins involved in transcriptional regulation, such as histone deacetylases (PF3D7_1472200) and specific transcription factors (PF3D7_1222400, PF3D7_1222600) from the ApiAP2 family were globally less expressed in the transgenic back-mutant $7 \mathrm{G} 8^{\text {pfcrt_ }}{ }^{T 76 K}$ and control $7 \mathrm{G} 8^{\text {pfcrt_CTL }}$ strains in comparison to the parental line 7G8 (Fig. 5a, b, Additional file 15: Table S9A,B). Additionally, genes involved in the maintenance of mitochondrial integrity and activity, such as the mitochondrial import inner membrane translocase subunits TIM13 and TIM44 (PF3D7_1242900 and PF3D7_1125400, respectively) and one of the cytochrome $\mathrm{c} 1$ precursors (PF3D7_1462700), showed 2- to 3.5-fold lower transcript levels in $7 \mathrm{G} 8^{\text {pfcrt_CTL }}$ and $7 \mathrm{G} 8^{\text {pfrt_t } T 76 K}$ in comparison to 7 G8 (Fig. 5a, b, Additional file 15: Table S9A,B). Stagespecific analysis revealed that down-regulation of the genes encoding the TIM subunits was most apparent during the schizont stage for both transgenic strains (Additional files 16 and 17: Tables S10E and S11E respectively), which shared many of the genes displaying a decreased expression when compared to 7G8 (Fig. 5c, Additional file 15: Table S9C). On the other hand PF3D7_0609400, which encodes a 


\section{a}

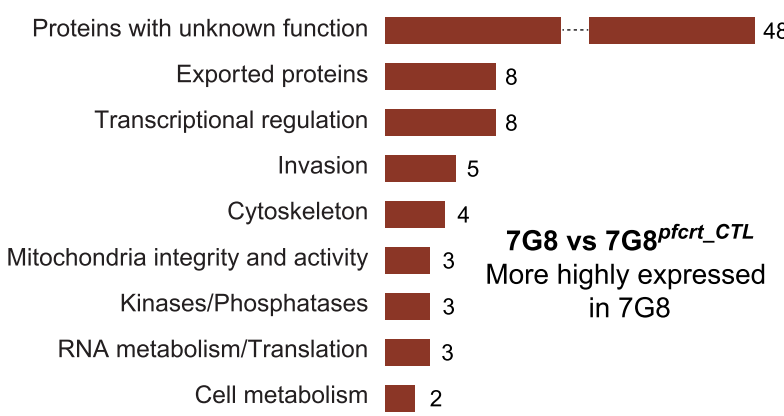

Post-translational modifications 1

Lipid biosynthesis 1

C

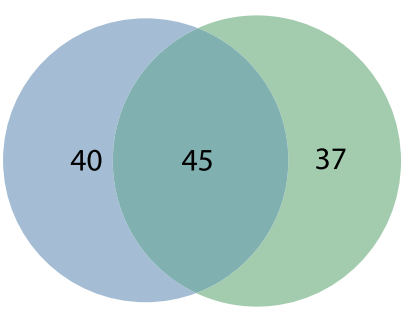

More highly expressed in 7G8 (Fold change $>1.5$ ) b

Proteins with Unknown Function

Transcriptional regulation

Invasion

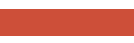

41

Cell metabolism

Exported proteins

7

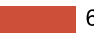

Protein Folding/Turnover

Mitochondrial activities

Lipid synthesis

Cytoskeleton

4

$7 \mathrm{G} 8$ vs $7 \mathrm{G}^{\text {pfcrt_t76K }}$

More highly expressed

Protein/Enzyme regulation 2

Translation 2

Ion transport 1

Phosphatases/Kinases 1
$7 \mathrm{G} 8$ vs $7 \mathrm{G}^{\text {pfort_CTL }}$

$7 \mathrm{G} 8$ vs $7 \mathrm{G} 8^{\text {pfort_t } 776 K}$

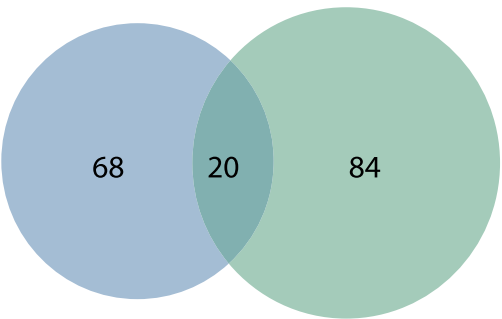

More lowly expressed in 7G8

(Fold change < 0.6) d

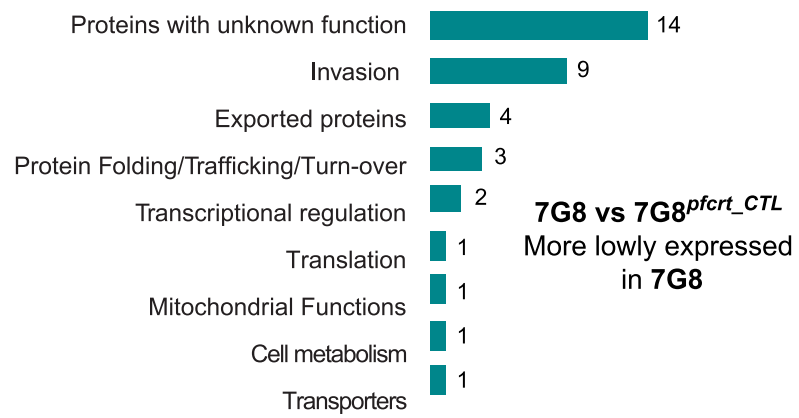

Proteins with unknown function

Invasion

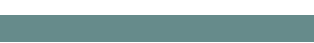
21 Exported proteins $\quad 4$ Protein Folding/Turn-over $\quad 4$

Translation 4

Transporters 2

Protein trafficking 2

Phosphatases/Kinases 2

$7 \mathrm{G} 8$ vs $7 \mathrm{G} 8^{\text {pfcrt_T76K }}$ More lowly expressed in $7 \mathrm{G} 8$

Protein signaling 1

Lipid biosynthesis 1

Mitochondrial functions 1

Fig. 5 Genes with significant expression fold changes organized by categories for pairwise comparisons of the transcriptomes in the pfcrt data set. Gene expression fold changes were calculated as the difference between the areas under the curve generated on the full 384 time point data and were then normalized using a baseline created by the background pool of 11 transcriptomes. For each gene, all 55 possible pairwise combinations of transcriptomes created a distribution of expression fold changes, which was used as a normalization factor. To identify significant differences, we applied a threshold of a fold change $>1.5$ and a normalized fold change at least 2 standard deviations (SD) above the mean for higher gene expression, and a threshold of a Fold change $<0.6$ and a normalized fold change 3 SD below the mean for lower expression. a Genes with higher expression in the parental line 7G8 in comparison to the recombinant control strain 7G8 ${ }^{\text {ffrt_CTL}}$. $\mathbf{b}$ Genes showing higher expression in $7 \mathrm{G} 8$ compared to the recombinant back-mutant strain $7 \mathrm{GG} 8^{\text {pfcrt_T76K }}$. $\mathbf{c}$ Venn diagrams depicting the overlap of genes with higher (fold change $>1.5$, left) or lower (fold change $<0.6$, right) expression in $7 \mathrm{G} 8$ for both $7 \mathrm{G} 8$ vs. $7 \mathrm{GG} 8^{\text {pfcrt_CTL }}$ and $7 \mathrm{G} 8$ vs. $7 \mathrm{G} 8^{\text {pfrr_t } 776 \mathrm{~K}}$ pairwise comparisons. d Genes with lower expression in comparison with the recombinant control strain 7G8 ${ }^{\text {pfrt_c } C L}$. e Genes with lower expression in the parental line $7 \mathrm{G} 8$ in comparison with the recombinant back-mutant strain $7 \mathrm{G} 8^{\text {pfrt_ } 776 \mathrm{~K}}$ 
cardiolipin synthase equally central to the mitochondrial inner membrane, was among the limited number of genes with higher transcript levels in both transgenic strains (Fig. 5c), particularly at the trophozoite stage (Additional files 16 and 17: Tables S10D and S11D). These observations suggest that decreased pfcrt expression, regardless of its haplotype in the 7G8 background, might affect mitochondrial integrity and functions.

When examining the $p f c r t$-modified transgenic strains, we observed increased expression in several genes encoding putative proteins involved in protein folding, trafficking and turnover (Fig. $5 \mathrm{~d}, \mathrm{e}$ ). These include heat shock and DnaJ proteins, which were on average 2-fold more highly expressed in comparison to 7G8 (Additional file 15: Table S9D-F). Increased expression was also observed with pfhrp3 (PF3D7_1372200), whose product is hypothesized to be involved in hemozoin formation in the parasite digestive vacuole [58]. These observations were confirmed by HATS (Fig. 6), which provided evidence for higher expression of genes in the categories of chaperones and protein catabolism in both $7 \mathrm{G}^{\text {pfcrt_CTL }}$ and $7 \mathrm{~GB}^{\text {pfcrt_T76K }}$ (Fig. 6a, b). Interestingly, there was a substantial overlap between the gene sets depleted in $7 \mathrm{G} 8$ in comparison to either $7 \mathrm{G} 8^{\text {pfcrt_CTL }}$ or $7 \mathrm{G}^{\text {pfcrt_T76K }}$ (Fig. 6c). Altogether these results suggest that pfcrt transcript levels can influence both parasite-specific processes and general biological pathways irrespective of this transporter's drug resistance phenotype (see Discussion).

Other genes whose expression was higher in the transgenic 7G8 strains in comparison to the parental line were mostly involved in parasite invasion and encoded merozoite surface proteins as well as glideosome and rhoptry components (Figs. 5d, e, 6a, b). Interestingly, these effects were accentuated in the back-mutant $7 \mathrm{G}^{\text {pfcrt_T76K }}$ at both the individual gene as well as gene set levels, during most of the parasite IDC (Fig. 7a-e, Additional files 15 and 16: Table S9 and S10 respectively).

We also compared the transcriptional profiles of $7 \mathrm{G}^{\text {pfcrt_T76K }}$ and $7 \mathrm{G} 8^{\text {pfcrt_CTL }}$ to assess the impact of the CQ resistance phenotype defined by PfCRT K76T. Genes differentially expressed between these lines were enriched for functions associated with DNA/RNA metabolism and protein catabolism (Fig. 7a, b). For instance, multiple genes encoding proteins possibly involved in DNA replication, such as mini-chromosome maintenance proteins (PF3D7_1355100, PF3D7_1417800), DNA helicases (PF3D7_0918600, PF3D7_1227100, PF3D7_ 1010200), a nucleotide excision repair enzyme (PF3D7_ 0710400) and the DNA polymerase $\alpha$ subunit (PF3D7 1463300) were $\sim 2$-fold more highly transcribed in 7G8 $8^{\text {pfrt_CTL }}$ (Fig. 7a, Additional file 15: Table S9G). Higher expression of some of these genes was observed throughout the IDC as shown by stage-specific analysis (Additional file 18: Table S12). Transcripts involved in RNA processing such as components of the exosome complex (PF3D7 0720000, PF3D7_0209200) showed 1.5 to 3-fold higher levels in $7 \mathrm{G}^{\text {pfcrt_C}}{ }^{-} T L$, mainly during the ring and trophozoite stages (Additional file 18: Table S12A,C,G). These observations were confirmed by HATS analysis (Fig. 7c, d).

Expression analysis of individual genes and gene sets indicated that genes involved in translation and protein stability, trafficking and turnover, such as those encoding ribosomal subunits and protein chaperones, respectively were also more highly transcribed in the transgenic control line 7G8 $8^{\text {pfcrt_CTL }}$ (Fig. 7a, c, Additional file 15: Table S9G). Stage-specific analysis demonstrated that higher expression of some of these genes was detected throughout the entire IDC (Additional file 18: Table S12K). Furthermore, HATS revealed that the categories of aminoacyl-tRNA ligases and synthetases were depleted in the back-mutant strain in comparison to both parental and control lines (Figs. 6b and 7c), although several individual genes within these categories, including some encoding a glutamate-tRNA ligase (PF3D7_1349200 and PF3D7_ 1357200), displayed higher expression in $7 \mathrm{G} 8^{\text {pfcrt_T76K }}$ at either ring or schizont stages (Additional file 18: Table S12B,F). Interestingly, HATS showed that gene sets linked to tRNA aminoacyl biosynthesis were also enriched in $7 \mathrm{G} 8^{\text {pfcrt_CTL }}$ in comparison to the parental strain (Fig. 6b). Several tRNA-ligases (PF3D7_0509600, PF3D7_1349200) and ribosyltransferases (PF3D7_1434100) also displayed higher transcript levels in a stage-specific manner (Additional file 16: Table S10B,J), suggesting that translation may also be affected when mutant $p f c r t$ expression is lowered (Additional file 19: Figure S7). Pathways linked to proteasome-mediated degradation and ribosome biogenesis were among the multiple genes and gene sets depleted in both control and parental strains in comparison to the back-mutant $7 \mathrm{G} 8^{\text {pfcrt_T76K }}$ (Figs. 5e, 6b and 7b, d, Additional files 15: Tables S9K,L). Altogether these observations indicate that the acquisition of drug-resistance mutations in pfcrt may impact protein synthesis and turnover pathways.

Expression analysis for individual genes identified several transporters in addition to $p f c r t$ that were differentially expressed between the two transgenic strains (Fig. 7a, b, Additional file 15: Table S9I,J), mostly in a stage-specific manner (Additional file 18: Table S12). This finding suggests a possible crosstalk between $p f c r t$ and other transporters. For example, $\sim 2$-fold higher transcript levels of the ABC transporter PF3D7_0813700 and the folate transporter FT1 (PF3D7_0828600) were detected in 7G8 $8^{\text {pfcrt_CTL }}$ (Additional file 18: Table S12A,G), while genes encoding amino acid (PF3D7 0629500) and ABC transporters (PF3D7_1229100 and PF3D7_1352100) were $~ 1.5$-fold more expressed in the back-mutant during either the ring or trophozoite stages (Additional file 18: Table S12D,F). 


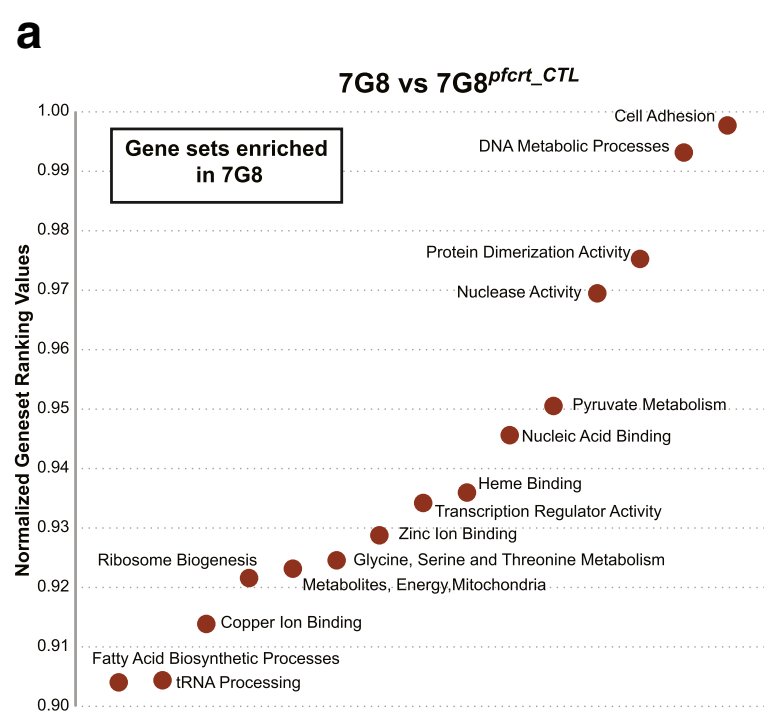

\section{$7 \mathrm{G} 8$ vs $7 \mathrm{G} 8^{\text {pfcrt_CTL }}$}

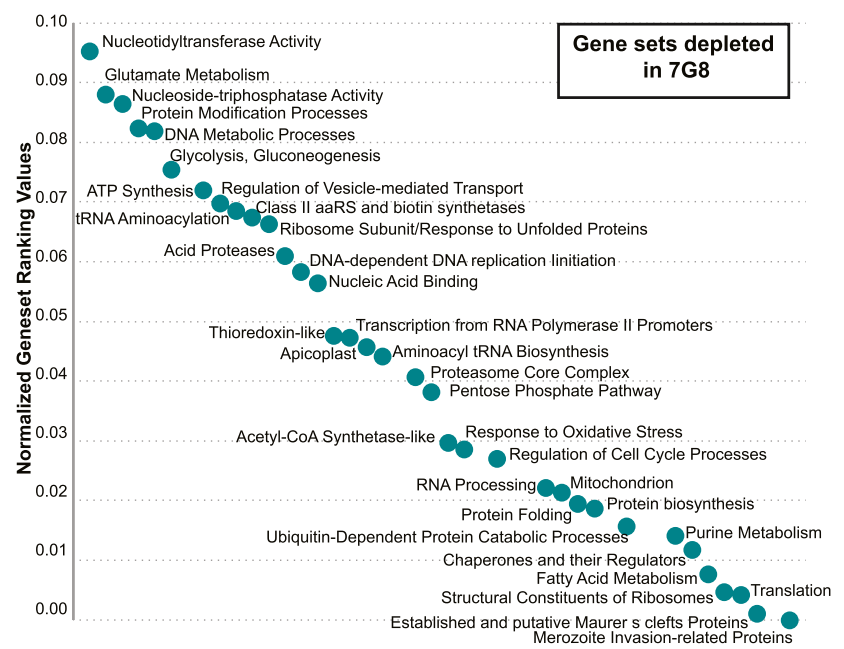

b

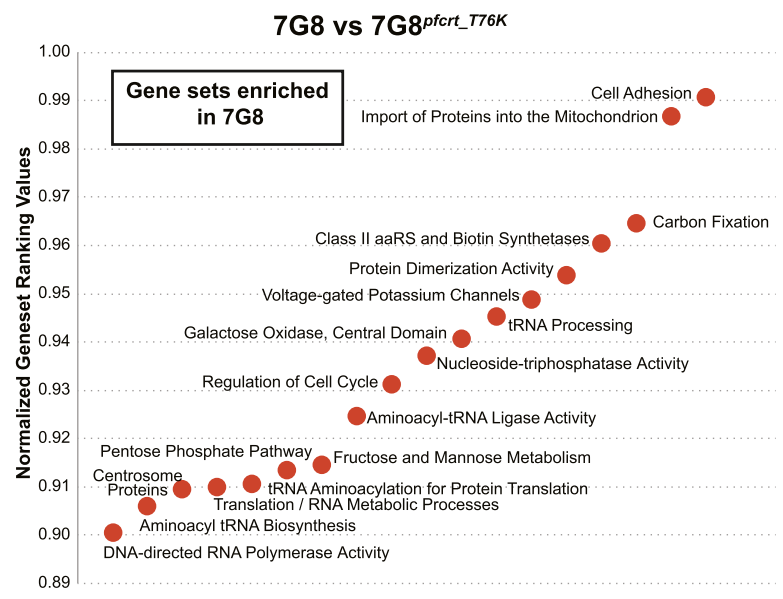

$7 G 8$ vs $7 G 8^{\text {pfort_ } 776 K}$

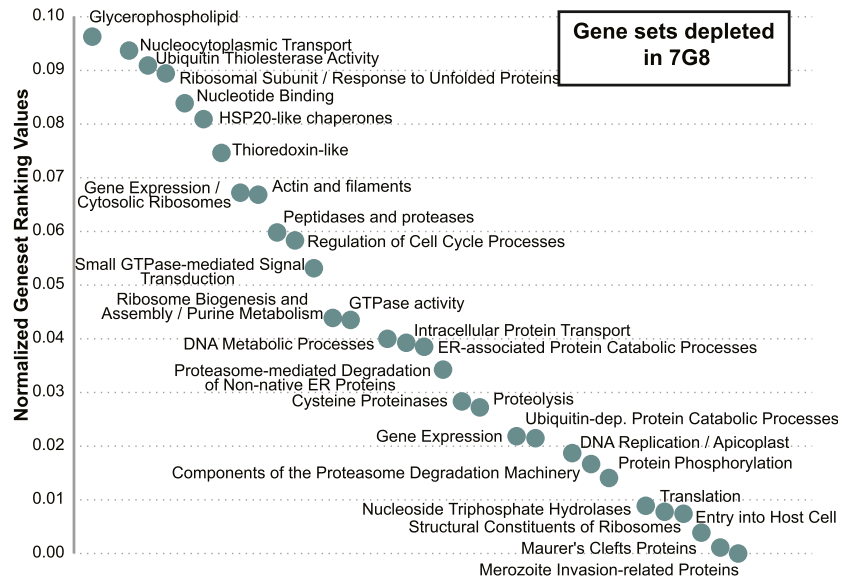

C

Gene sets enriched in 7G8 (Mean ranking > 0.9)

Gene sets depleted in $7 \mathrm{G8}$ (Mean ranking $<0.1$ )

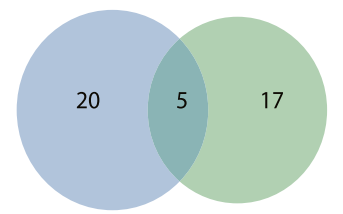

$7 G 8$ vs $7 G 8^{\text {Pfrt_c } C T L}$

$7 \mathrm{G} 8$ vs $7 \mathrm{G} 8^{\text {pfort_t } T 76 K}$

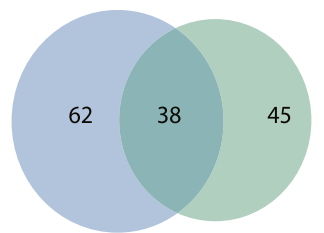

Fig. 6 Hypergeometric Analysis of Time Series (HATS) for pairwise comparisons of the transcriptomes in the pfcrt data set. As for the pfmdr 1 dataset, we performed HATS analysis by combining FFT to align the time series with a hypergeometric distribution approach based on random permutations to compute an enrichment score for each gene set. Normalization of the enrichment scores was performed using the expression baseline constituted by the background pool of 11 transcriptome data sets. As before, we applied a threshold of normalized gene set rank $>0.9$ for gene sets we considered positively enriched and normalized gene set rank $<0.1$ for gene sets considered negatively enriched. The gene sets were built using GO, KEGG, and the Malaria Parasite Metabolic Pathway. a Gene sets that are enriched (left, mean ranking values $>0.9$ ) or depleted (right, mean ranking values $<0.1$ ) in $7 \mathrm{G} 8$ in comparison with $7 \mathrm{G} 8^{\text {pfcrt_c } c L}$. b Gene sets that are enriched (left, mean ranking values $>0.9$ ) or depleted (right, mean ranking values $<0.1$ ) in $7 \mathrm{G} 8$ in comparison to $7 \mathrm{G} 8^{\text {pfrt_ } T 76 \mathrm{~K}}$. c Venn diagrams depicting overlaps of gene sets significantly enriched (left) or depleted (right) in $7 \mathrm{G} 8$ for both $7 \mathrm{G} 8$ vs. $7 \mathrm{G} 8^{\text {pfcrt_CTL }}$ and $7 \mathrm{G} 8$ vs. $7 \mathrm{G} 8^{\text {pfcrt_T76K }}$ pairwise comparisons 
a

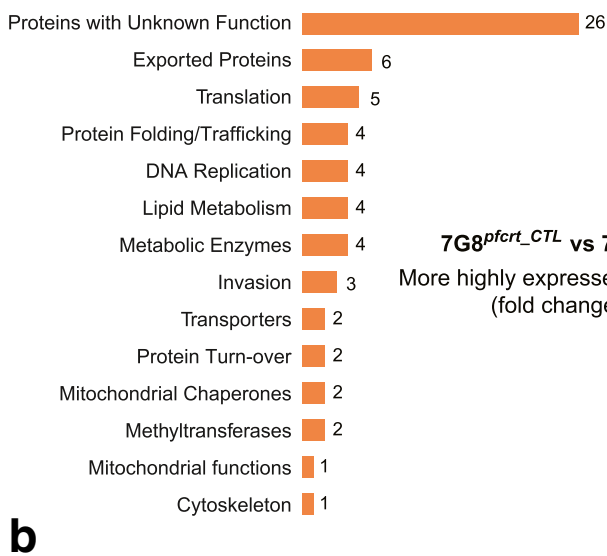

e

$7 \mathrm{G} 8^{\text {pfcrt_CTL }}$ vs $7 \mathrm{G} 8^{\text {pfcrt_T76K }}$

Ring

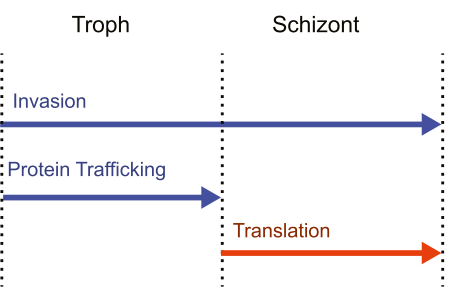

C

$7 G 8^{\text {pfcrt_CTL }}$ vs $7 \mathrm{G}^{\text {pfcrt_T76K }}$

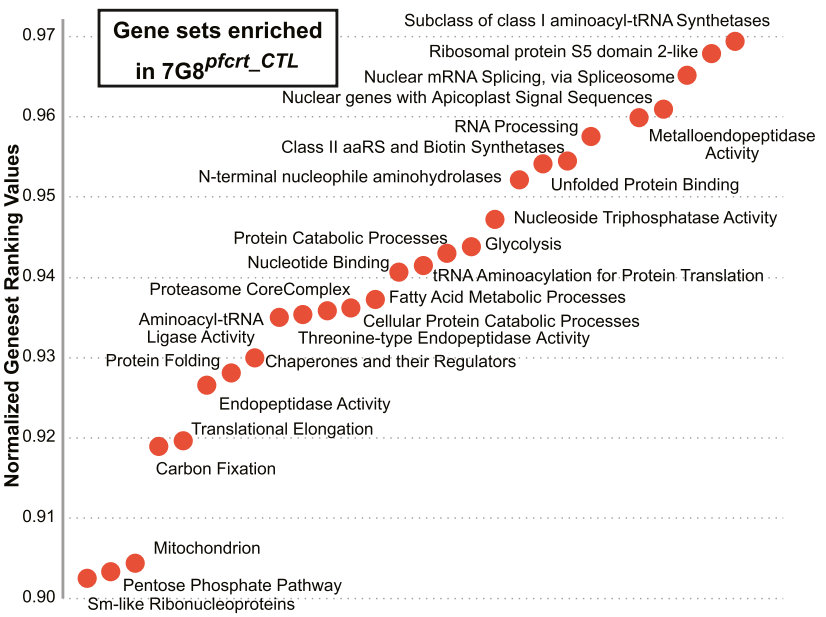

d

$7 \mathrm{G} 8^{\text {pfrrt_cTL }}$ vs $7 \mathrm{G}^{\text {pfcrt_T76K }}$

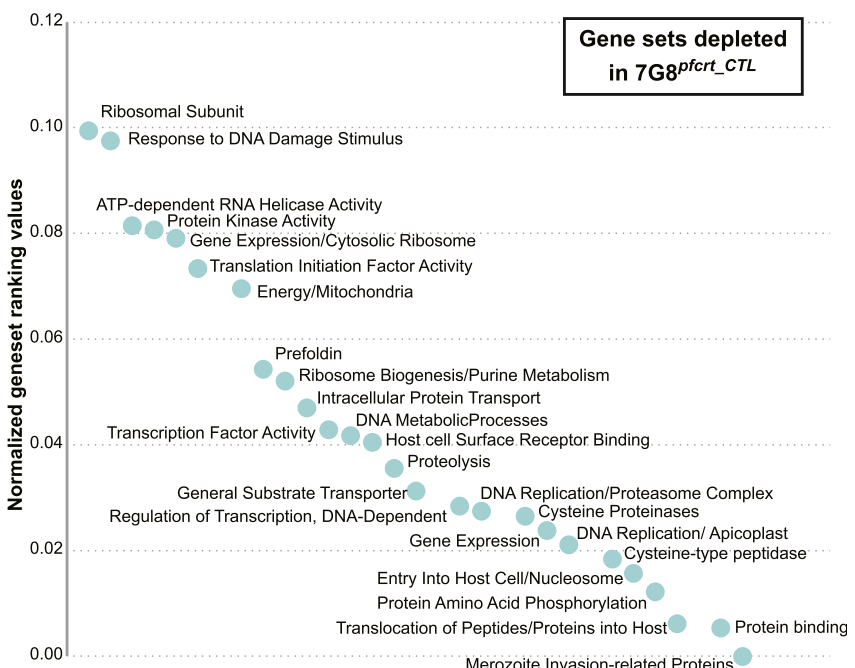

Fig. 7 Analysis of the transcriptional differences between $7 \mathrm{G} 8^{\text {pfcrt_CTL }}$ and $7 \mathrm{G} 8^{\text {pfrt_t T76K }}$. a Genes showing higher expression (fold change $>1.5$ and normalized fold change $\geq 2 \mathrm{SD}$ above the mean) in the recombinant control $7 \mathrm{G} 8^{\text {pfrt_c } C L}$ in comparison to the recombinant back-mutant strain $7 \mathrm{G} 8^{\text {pfcrt_T76K }}$. Gene expression fold changes were calculated as the difference between the areas under the curve generated on the full 384 time point data and were then normalized using a baseline created by the background pool of 11 transcriptomes. $\mathbf{b}$ Genes showing a lower

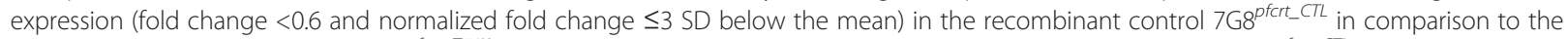
recombinant back-mutant strain 7G8 ${ }^{\text {pfcrt_T76K }}$. c HATS analysis was used to identify gene sets that are enriched in 7 G $8^{\text {pfrrt_cTL }}$ in comparison to $7 \mathrm{G} 8^{\text {pfcrt_T76K }}$ (mean ranking values $>0.9$ ). Enrichment scores were normalized using the expression baseline constituted by the background pool of 11 transcriptome data sets. $\mathbf{d}$ Gene sets that are depleted in $7 \mathrm{G} 8^{\text {pfrt_CTL }}$ in comparison to $7 \mathrm{G} 8^{\text {pfrt_T76K }}$ (mean ranking values $<0.1$ ), as identified from HATS analysis. e Stage-associated enrichment analysis. Gene sets with significant enrichment (orange) or depletion (blue) in $7 \mathrm{G} 8^{\text {pfcrt_cTL }}$ in comparison to $7 \mathrm{G}^{\text {pfrrt_T76K }}$ for three windows of the parasite IDC corresponding to rings ( $\mathrm{R}, 12-16 \mathrm{~h}$ post-invasion), trophozoites (T, 24-28 $\mathrm{h}$ post-invasion) and schizonts (S, 32-36 h post-invasion) using stage-enrichment analysis

\section{Comparison of gene expression patterns across all profiled transcriptomes reveals a transcriptional network that encompasses both pfcrt and pfmdr1}

Our comparative analysis of the transcriptome profiles of the FCB- and 7G8-derived parasite lines demonstrated that mutations and/or altered transcript levels in $p f m d r 1$ or $p f c r t$ impacted the expression of similar gene categories. We therefore assessed whether a transcriptional association between $p f c r t$ and $p f m d r 1$ might account for the similarities we observed between the transcriptome data sets.

Using the publicly available transcriptome data sets of the P. falciparum strains 3D7, Dd2, and HB3 [46, 48] 
that we added to our own data sets, we first performed an expression-based hierarchical clustering of the data, using PCC distances between gene pairs computed across all possible pairwise comparisons of transcriptomic profiles. $p f c r t$ and $p f m d r 1$ ranked closely to each other, and formed two distinguishable clusters with high scores for gene pair correlations (Fig. 8a). One of these clusters included a V-type ATPase localized in the parasite digestive vacuole (PF3D7_1354400, PCC 0.7-0.8), a DnaJ chaperone protein (PF3D7_1307200, PCC 0.70.8 ) and a transcription factor of the ApiAP2 family (PF3D7_0516800) (Additional file 20: Table S13). The other cluster contained the RNA Polymerase I responsible for rRNA transcription (PF3D7_1134700, PCC 0.8) (Additional file 21: Table S14). Interestingly, the two clusters also contained PF3D7 0823300 that encodes the histone acetyltransferase GCN5 and PF3D7_0303700, a gene involved in lipoamide synthesis, which is in concordance with a recent study reporting the coexpression of pfcrt with lipoamide synthesis genes [59]. Altogether these observations suggest an association between $p f m d r 1$ and pfcrt expression and that of genes with specialized functions in the digestive vacuole, in addition to genes having more general functions such as rRNA transcription and protein translation.
To further investigate the transcriptional association between $p f c r t$ and $p f m d r 1$, we conducted an additional hierarchical clustering of gene expression, this time, by ordering genes based on the level of correlation they displayed with either $p f c r t$ or $p f m d r 1$ expression (Additional file 22: Figure S8). While more than a hundred genes showed a high transcriptional correlation $(\mathrm{PCC}>0.7$ ) with each gene, we also detected a large number of genes that were strongly anti-correlated (PCC <-0.7) (Additional file 23: Table S15). Interestingly, many genes $(\sim 70 \%)$ with strong positive or negative transcriptional correlation with $p f c r t$ were additionally strongly correlated with pfmdr1 expression (Fig. 8b). A dozen of those genes encode transporters, components of the digestive vacuole, or redox enzymes (Table 2). Others were linked to DNA/RNA-dependent processes, translation, and protein trafficking (such as components of the PTEX parasite translocon of exported proteins) (Additional file 24: Table S16A). On the other hand, several genes implicated in either RNA processing or protein catabolism, such as those encoding splicing factors and elements of the proteasome complex, respectively, showed a strong anti-correlation with both $p f c r t$ and $p f m d r 1$ expression (Table 2, Additional file 24: Table S16B). These findings suggest the existence of a transcriptional network that encompasses both genes and evoke a possible

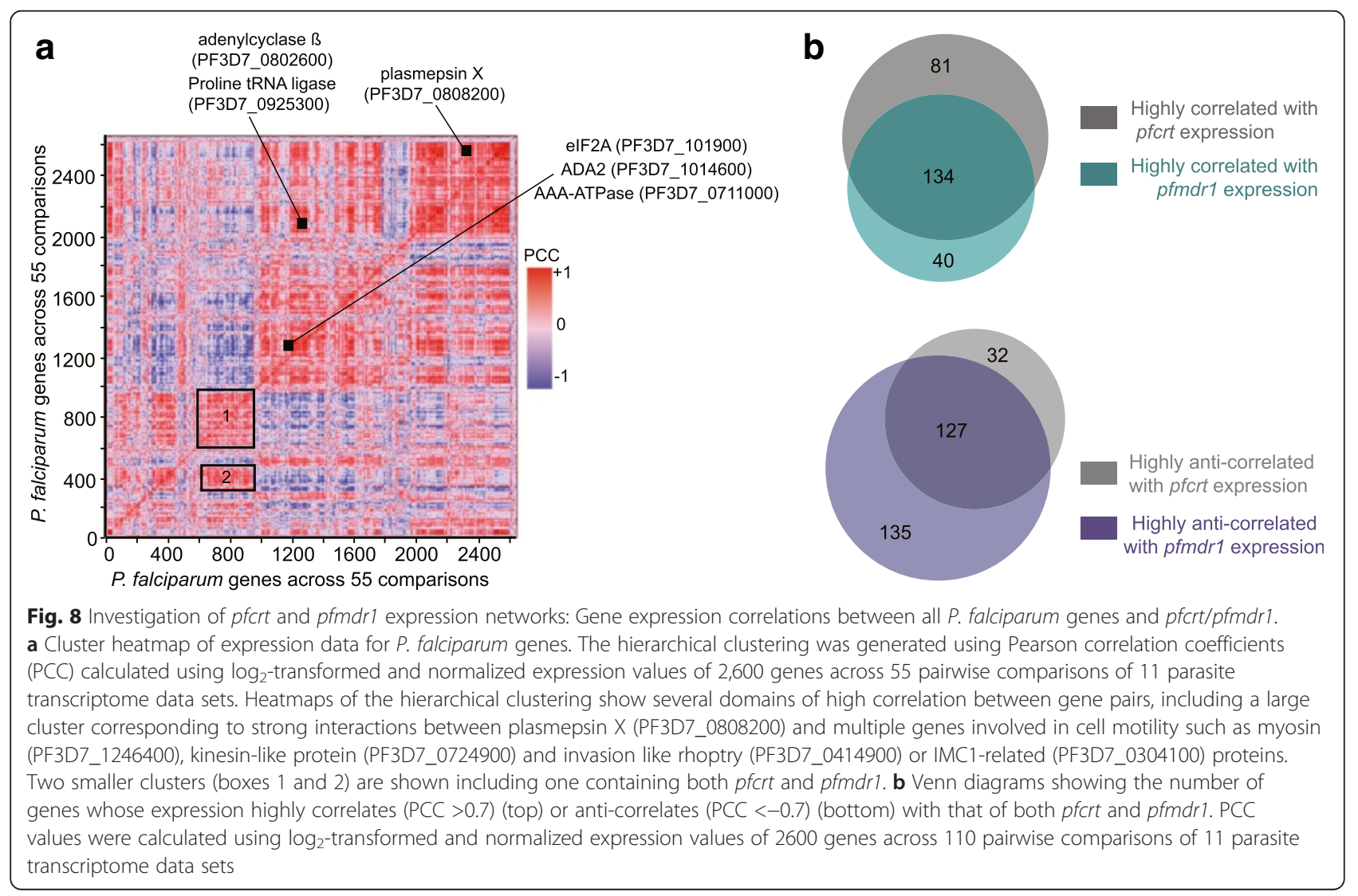


functional association between these two drug resistance transporters.

\section{Discussion}

Studies have previously defined the malaria parasite transcriptome as hard-wired, capable only of very limited changes at the transcript level in response to specific metabolic challenges [43, 44, 60]. Nevertheless, more recent studies have predicted gene functions and networks using genome-wide transcriptional profiling of $P$. falciparum growth perturbations, and have provided evidence that the parasite can display transcriptional changes even if of low amplitude in response to chemical challenges [61]. In the present study, we have addressed how variant forms of two primary determinants of parasite drug susceptibility can affect the asexual blood stage transcriptome. This focused on a comparative analysis of the transcriptome profiles of $P$. falciparum parasite lines engineered to display distinct levels of expression and/or mutation in the genes coding for the drug resistance transporters PfCRT and PfMDR1. Genome-wide analysis of the transcriptomes was performed both at the gene and gene set levels using computational approaches that identified and excluded transcriptional differences due to stochastic expression. This enabled us to define a transcriptional baseline and

Table 2 Table summarizing categories of genes with strong positive (PCC >0.7) and negative correlation (PCC $<-0.7)$ of expression with that of both pfcrt and pfmdr1. Genes correlated or anticorrelated with pfcrt and pfmdr1 transcript levels

\begin{tabular}{|c|c|c|}
\hline Categories & Gene ID & Description \\
\hline \multicolumn{3}{|l|}{ Positively Correlated with pfcrt and pfmdr1 } \\
\hline \multirow[t]{10}{*}{ Transporters/Components of the digestive vacuole } & PF3D7_0519200 & vacuolar ATP synthetase \\
\hline & PF3D7_0614300 & organic anion transporter \\
\hline & PF3D7_0810200 & $A B C 1$ family, putative ( $A B C K 1)$ \\
\hline & PF3D7_1132500 & amino acid transporter, putative \\
\hline & PF3D7_1354400 & V-type ATPase, putative \\
\hline & PF3D7_1456800 & V-type $\mathrm{H}($ )-translocating pyrophosphatase, putative \\
\hline & PF3D7_1129900 & transporter, putative \\
\hline & PF3D7_1454400 & aminopeptidase $P$ \\
\hline & PF3D7_1126000 & threonine-tRNA ligase (ThrRS) \\
\hline & PF3D7_1332800 & translation initiation factor 6 , putative \\
\hline \multirow[t]{4}{*}{ Translation } & PF3D7_1367700 & alanine-tRNA ligase (AlaRS) \\
\hline & PF3D7_1445100 & histidine-tRNA ligase, putative \\
\hline & PF3D7_1204300 & eukaryotic translation initiation factor 5A (EIF5A) \\
\hline & PF3D7_0422500 & pre-mRNA-splicing helicase BRR2, putative (BRR2) \\
\hline \multirow[t]{2}{*}{ RNA metabolism } & PF3D7_0623900 & ribonuclease $\mathrm{H} 2$ subunit $\mathrm{A}$, putative \\
\hline & PF3D7_1421400 & DNA-directed RNA polymerase III subunit C, putative \\
\hline \multicolumn{3}{|l|}{ Negatively Correlated with pfcrt and pfmdr1 } \\
\hline \multirow[t]{6}{*}{ DNA/RNA metabolism } & PF3D7_1125300 & DNA-dependent RNA polymerase \\
\hline & PF3D7_0105900 & DNA binding protein, putative \\
\hline & PF3D7_0617800 & histone $\mathrm{H} 2 \mathrm{~A}$ \\
\hline & PF3D7_1033600 & Myb2 protein \\
\hline & PF3D7_0716100 & large ribosomal subunit assembling factor, putative \\
\hline & PF3D7_0420400 & ribosome recycling factor, putative \\
\hline \multirow[t]{3}{*}{ RNA processing/Translation } & PF3D7_0610100 & step II splicing factor, putative \\
\hline & PF3D7_1446900 & glutaminyl-peptide cyclotransferase, putative \\
\hline & PF3D7_0904600 & ubiquitin specific protease, putative \\
\hline \multirow[t]{2}{*}{ Protein Catabolism } & PF3D7_0815700 & ubiquitin \\
\hline & PF3D7_0413600 & $26 \mathrm{~S}$ proteasome AAA-ATPase subunit RPT3, putative \\
\hline \multirow[t]{2}{*}{ Transporters } & PF3D7_1303500 & sodium/hydrogen exchanger, $\mathrm{Na}, \mathrm{H}$ antiporter \\
\hline & PF3D7_1352100 & ABC transporter, (heavy metal transporter family), putative \\
\hline
\end{tabular}


detect genes whose expression significantly diverged, implicating them as candidate factors in a parasite transcriptional adaptation to the $p f c r t$ and $p f m d r 1$ changes that were genetically introduced.

Among the $~ 5,500$ coding genes in $P$. falciparum genome, we surveyed the expression changes for a set of 3,000 to 3,500 genes for which the microarray data passed quality inspection across at least 6 of the 8 time points in the series. It is therefore possible that we missed additional genes with differential transcriptional profiles. Despite this limitation, we found that genetic modifications of the loci coding for these two digestive vacuole transporters notably affect the transcription of genes coding for RNA-binding proteins, transcription factors, elements of the translation initiation and elongation machinery, and components of both the spliceosome and proteasome complexes. This observation was particularly striking in the $p f m d r 1$ data set that included the FCB isogenic strain, $\mathrm{FCB}^{\text {ffmdrl }}$ 3' $^{\prime} K D$. Deamplification of a large region on chromosome 5 that encompassed pfmdr1 led to the increased expression of genes involved in translation and reduced levels of transcripts implicated in protein catabolism and modifications. This represented in total more than $10 \%$ of the global expression changes and is concordant with expression Quantitative Trait Loci analyses that previously demonstrated an association between the pfmdr1-containing amplicon and both post-translational modifications and protein catabolic processes [62]. In agreement with that study [62], we identified a large number of genes involved in proteasome-mediated degradation and protein ubiquitination that display differential expression between FCB and FCB ${ }^{p f m d r 1}{ }^{3}{ }^{\prime} K D$. This suggests that amplification of the $p f m d r 1$-containing amplicon can be accompanied by a global reduction of DNA and RNA metabolic activities, and increased protein catabolism.

Our study also identified mechanisms of transcriptional down-regulation across the pfmdr1 amplicon in both 106/1 (that carries two physical copies) and $\mathrm{FCB}^{\text {pfmdr1_3'KD }}$ (whose second pfmdr1 copy had been disrupted, and for which the expression of adjacent genes also became downregulated). Earlier studies of Thai field isolates had shown considerable variability in both pfmdr1 copy number and amplicon size, which would impact expression levels of both pfmdr1 and its neighboring genes [34]. Our data highlight the need to account for expression when assessing the association between pfmdr1 copy number and drug susceptibility states, and underscore the potential for additional biological differences resulting from neighboring loci.

Differential expression of genes with a role in fundamental biological processes such as RNA translation, ubiquitin-dependent catabolic processes and protein folding/trafficking was also observed in the pfcrt data set, albeit to a lesser extent. Gene sets associated with these pathways showed a greater enrichment in both 7G8-derived transgenic strains that were characterized by a decreased $p f c r t$ transcript level, in comparison to the parental 7G8 line. This suggests an association between the degree of $p f c r t$ expression and the regulation of cellular protein levels, regardless of PfCRT haplotype.

Intriguingly, levels of pfcrt transcripts, irrespective of the transporter polymorphism, also impacted sets of genes implicated in maintaining mitochondrial integrity and function, as indicated by the significant decrease in expression of genes coding for multiple subunits of the translocase complexes of the mitochondria inner membrane [63]. In contrast, the transgenic strains that differ in the nature of the residue at position 76 in PfCRT displayed a differential enrichment of gene sets associated with amino-acyl tRNA synthases and ligases. Several of the genes coding for these elements, which play a central role in RNA translation, were more highly expressed in a stage-specific manner in the transgenic control in comparison to the back-mutant strain. These findings suggest that the impairment of PfCRT transport function through the acquisition of resistance mutations might impact the cellular level of amino acids. This hypothesis is consistent with the observation that PfCRT polymorphisms affect hemoglobin catabolism and that CQresistant parasites such as 7G8 are characterized by a phenotype of excessive peptide accumulation, which might in turn impact the reserve of amino acids available for protein synthesis [64]. Additionally, the differential expression of genes encoding putative transporters between the two transgenic strains suggest that PfCRT resistance mutations might more generally affect intracellular transport dynamics, possibly as a secondary effect of the peptide accumulation phenotype.

Interestingly, both $p f m d r 1$ and $p f c r t$ transcriptome data sets displayed differences in the level of expression of genes coding for invasion proteins. Higher transcript levels of rhoptry and merozoite surface proteins, or components of the glideosome were detected in parasite strains with either reduced $p f m d r 1$ expression such as $\mathrm{FCB}^{\text {pfmdr }{ }_{3}{ }^{\prime} K D}$ and $106 / 1$ or with a PfCRT wild-type haplotype such as the back-mutant strain $7 \mathrm{G}^{\text {pfcrt_T76K }}$. This, added to the observation that both CQ-sensitive strains $106 / 1$ and $7 \mathrm{G} 8^{p f c r t-T 76 K}$ had a shorter IDC than the other parasites, suggests an impact of these genetic changes on growth rates and parasite fitness [64]. Interestingly, while 106/1 and FCB have genome sequences that are almost identical [65] (suggesting a common lineage), our data indicate notable differences in both their PfCRT haplotype and their levels of expression from the pfmdr1 amplicon region. By comparing FCB, $\mathrm{FCB}^{\text {pfndr }{ }_{-}{ }^{\prime} K D}$ and $106 / 1$, we could disentangle the transcriptional changes due to lower expression/gene copy 
variation in the chromosome 5 amplicon from the effect of different pfcrt genotypes. For instance, 106/1 appears to express higher levels of ribosomal protein genes compared to both FCB strains, suggesting a response to its particular PfCRT haplotype that lacks the CQ resistance-conferring $\mathrm{K} 76 \mathrm{~T}$ mutation. On the other hand, our data suggest that the transcriptional downregulation of the pfmdr1-containing amplicon on chromosome 5 (in 106/1 and $\mathrm{FCB}^{\text {pfm }}{ }^{211_{-} 3^{\prime} K D}$ ) might affect parasite physiology, notably through the reduced expression of genes coding for multiple transporters and components of the parasite digestive vacuole, such as $\mathrm{ABC}$ and drug/metabolite transporters and V-type ATPases. Such an observation was particularly striking when performing a comparative analysis of the transcriptomes by stage, demonstrating how such an approach can complement analyses of gene expression averaged across time points.

Our investigations across multiple lines uncovered evidence of co-regulation of $p f c r t$ and $p f m d r 1$, which both also positively correlated across the genome with a suite of genes coding for transporters, components of the parasite digestive vacuole that include peptidases involved in hemoglobin catabolism, or redox enzymes. Conversely, pfcrt and pfmdr1 transcription anticorrelated with a set of genes encoding proteins involved in RNA processing, including splicing factors. Broader biological processes including transcriptional regulation, translation and post-translational processing contained genes that showed either positive or negative correlations with $p f c r t$ and pfmdr1. Overall this suggests the existence of an elaborate equilibrium in $P$. falciparum gene expression that maintains optimal states of parasite physiology that extend beyond the digestive vacuole.

Our study builds on the recent work from Siwo and colleagues who constructed $p f c r t$ co-expression networks using transcriptional measurements at the early trophozoite stage for the progeny of a genetic cross between CQ-sensitive and CQ-resistant parental strains [59]. Their analysis provided evidence that functional partners of both CQ-resistant and CQ-sensitive pfcrt include genes involved in hemoglobin catabolism, which agrees with our own observations. Additionally, the authors attributed divergence in co-expression networks between CQ-resistant and CQ-sensitive pfcrt to genes involved in DNA repair and histone acetylation. Interestingly, we found that expression of the histone acetyltransferase GCN5 (PF3D7_0823300) clustered with that of pfcrt and pfmdr1, further highlighting an association between the expression of these two transporters and general transcriptional processes.

Furthermore, our data extend prior observations of joint contributions of $p f c r t$ and $p f m d r 1$ polymorphisms to drug resistance phenotypes [22, 28, 66], including evidence of linkage disequilibrium, by now providing evidence of a strong correlation between pfcrt and pfmdr1 transcript levels. This has direct implications for drug resistance studies that measure $p f m d r 1$ copy number as a marker of decreased parasite susceptibility to antimalarial drugs [67] by implying an previously unrecognized role for accompanying changes in pfcrt.

\section{Conclusions}

PfCRT and PfMDR1 are two key determinants of $P$. falciparum parasite susceptibility to antimalarial compounds. Both are transporters localized in the intraerythrocytic digestive vacuole, whose primary functions in addition to carrying antimalarial drugs remain to date unknown. Our transcriptome profiling of various $P$. falciparum strains, whose differences in pfcrt or pdmrl coding sequence or copy number contribute directly to multidrug resistance, provide insight into how the acquisition of these genetic changes impact parasite physiology beyond the digestive vacuole. These changes include peptide catabolism, digestive vacuole homeostasis, RNA metabolism and protein synthesis. Our findings, based on the development of an analytical method that we term Hypergeometric Analysis of Time Series, also reveal a closely coordinated co-regulation of $p f c r t$ and pfmdr 1 transcript levels. Of particular interest now will be to further study how these transcriptional crosstalks affect downstream processes, in particular at the level of the parasite proteome and metabolome.

\section{Methods}

\section{Parasite culture}

All parasite lines described here were propagated under standard conditions [68] in RPMI-1640 medium with Lglutamine, supplemented with $50 \mathrm{mg} / \mathrm{l}$ hypoxanthine, $25 \mathrm{mM}$ HEPES, $0.25 \%$ sodium bicarbonate, $0.01 \mathrm{mg} / \mathrm{ml}$ gentamicin and $0.5 \%$ Albumax II (Invitrogen, Carlsbad, CA, USA). Parasites were synchronized by two consecutive sorbitol treatments timed $12 \mathrm{~h}$ apart for three or more successive generations and allowed to recover for another parasite asexual blood stage cycle before initiating time point samplings. For each time course, cultures were progressively expanded to a volume of 250 $300 \mathrm{~mL}$ at $3 \%$ hematocrit with 8 to $10 \%$ of tightly synchronous populations of late $P$. falciparum schizonts. Parasites were inoculated in a bioreactor (Applikon, Foster City, CA, USA) in the presence of fresh erythrocytes and allowed to reinvade for the next $2-3 \mathrm{~h}$ at high hematocrit $(8 \%)$. Gas and temperature conditions were monitored with a Bio Controller unit ADI 1030. The first time point of the series was determined by the peak of invasion, after which cultures were diluted to a final volume of $1,000 \mathrm{~mL}$ and $2 \%$ hematocrit. At least $90 \%$ 
of the parasites were in the early ring stage and in general, cultures reached a parasitemia of 10 to $20 \%$. Equal parasite samples were harvested every $6 \mathrm{~h}$ throughout the 48-hour IDC and immediately centrifuged. Pellets of parasitized erythrocytes were directly stored in Trizol $^{\circ}$ for subsequent RNA extraction and cDNA synthesis.

\section{RNA preparation, CDNA synthesis and DNA microarray hybridizations}

Total RNA was isolated and synthesis of first strand amino-allyl cDNA was performed as previously reported $[69,70]$ using $125 \mathrm{U}$ of Superscript II reverse transcriptase (Invitrogen, Carlsbad, CA, USA). Each reaction was concentrated on a Zymo DNA clean and concentrator-5 column (Zymo Research, Irvine, CA, USA) and labeled with Cy5 dye (GE Healthcare, Piscataway, NJ, USA). The reference RNA pool consisted of Cy3-coupled cDNA samples prepared from RNAs representing all developmental stages at 6-hour intervals of the IDC of the 3D7 line. Equal amounts of labeled samples from each time point and reference pool were subjected to array hybridization for $16-18 \mathrm{~h}$ at $65^{\circ} \mathrm{C}$ on 70 -mer P. falciparum microarray chips that contained 10,416 features and represented all 5,363 coding genes as previously reported [70]. Data were acquired using a GenePix 4000B scanner and analyzed with the GenePix Pro 4 software (Molecular Devices, Sunnyvale, CA, USA).

\section{Microarray data normalization and quality control}

Automatic definition of hybridization spots was refined by manual inspection, and acquired raw data were initially filtered to exclude poor quality spots. The Cy5 and Cy3 intensity channels were separately normalized using the mean difference between the median foreground intensity and the median background intensity of a set of representative probes. This set included probes that contained more than $50 \%$ of the pixels with intensities higher than 2 standard deviations above the background pixel intensity for both $\mathrm{Cy} 5$ and $\mathrm{Cy} 3$ signals, displayed at least 200 expression units and a regression correlation coefficient greater than or equal to 0.75 [48]. After background subtraction, the $\log _{2}$ of the $(\mathrm{Cy} 5 / \mathrm{Cy} 3)$ intensity ratio was extracted for each probe and gene expression ratios were computed using the median of the probe level values. For genes with values for at least 6 of 8 time points, missing values were imputed using linear model prediction based on PCC values and accounting for mean and standard deviation differences.

Transcriptomes of all lines were reconstituted using the FFT method $(f f t)$ function in R) to sort genes according to phase, and impute expression levels at 376 unassayed time points, one for each $7.5 \mathrm{~min}$ of the 48hour IDC. To correct for variations in developmental stage speed between parasite lines, dynamic time warping
[71] was applied to determine the optimal post-invasion time point (corresponding to the first sampling time point) along the IDC using the PCC between each pair of imputed points. Each parasite line was aligned using the 3D7 transcriptome as reference [46]. The alignments were confirmed by applying PCA. The first two principal components, derived from approximately 3,000 dimensions of expression data, accurately recapitulated the progression of the parasite lines throughout the IDC, while succeeding components focused on differences between parasite lines.

\section{Microarray data analysis}

To assess gene expression level differences between parasite lines, the area under the curve (AUC) of absolute gene expression plots was computed on the full 384 time point data and the fold change was calculated as the difference between AUCs in pairwise comparisons. OPLS-DA (Orthogonal Partial Least Square-Discriminant Analysis) was used to create disjoint PCA models for each parasite line [72] and exploit class-orthogonal variation, thereby permitting the identification of genes expressed out of phase. Both approaches resulted in a similar gene ranking with a Spearman correlation of 0.89. To determine significant variation between parasite lines, a cut-off of $>1.5$-fold difference in gene expression was assigned.

All values were normalized using a background pool constituted of 11 transcriptome data sets that constituted a baseline for gene expression fold change and gene set enrichment. In addition to that reported in the present study, the pool included two IDC transcriptome data sets generated for Dd2 parasites pressured longterm with pulses of dihydroartemisinin (the 3b1 line) and the non-pressured parent [73], as well as the transcriptome data set previously reported for the 3D7, Dd2 and HB3 strains [46, 48]. This background pool was used to generate 55 pairwise comparisons (or 110, if taking into account analysis in both directions) and create a distribution of fold changes for each analysed gene, which was then used as a normalizing factor. When comparing two lines, only the remaining nine were used to build the fold change distribution. The analysis was conducted using a threshold of two or three standard deviations above this mean fold change. This method, based on a large dataset of pairwise comparisons, enables us to identify even genes for which expression changes are of small amplitudes.

To analyze gene sets, we developed an algorithm that we termed Hypergeometric Analysis of Time Series (HATS). The source code and pseudocode describing HATS are provided in Additional file 25. HATS is based on GSEA [56] and consists in assigning a rank probability to each gene in a given gene set using GSEA and 
then applying hypergeometric distribution instead of a weighted Kolmogorov-Smirnov-like statistic [56] to evaluate the enrichment score. To this end, 1,000 random permutations of the data were generated, and on each of these permutations we calculated gene rank probabilities $P$ for each gene set. These probability values were averaged per gene set across the 1,000 permutations and used as a scaling factor defined as $-\log _{10}(P)$ to generate a corrected enrichment score from that originally computed by GSEA. The false discovery rate (FDR) was estimated by comparing the distributions of the actual enrichment scores to those derived from the random permutations. Noise reduction was achieved by forcing all FDR values to monotonically decrease at the tails. Finally, theses results were then normalized by comparing them to the pool of 11 transcriptome datasets, across which pairwise GSEA comparisons were conducted for all possible combinations. Means and standard deviations of the pool-derived enrichment scores for each gene set were computed and used to generate normalized enrichment scores and remove sets that were stochastically expressed. We applied a cut-off for normalized gene set ranks of 0.1 for negatively enriched gene sets and of 0.9 for positively enriched gene sets.

Results were also analyzed by parasite developmental stages, using time windows corresponding to ring, trophozoite, and schizont stages [74]. Stage-associated enrichment was calculated by averaging gene expression across each developmental stage using the AUC derived from the imputed 384-time point curve and then performing GSEA. As before the enrichment scores were calculated using the hypergeometric distribution and the values were normalized using the background pool of transcriptome datasets.

Gene sets were assembled from gene ontology (GO) annotations [75], the Malaria Parasite Metabolic Pathway [76, 77], KEGG [78] and InterPro [79] databases, and literature searches. A summary of these gene sets is provided in Additional file 24: Table S16. We extracted the GO categories for $P$. falciparum from PlasmoDB (www.plasmoDB.org) such that 1,664 GO categories involving 4,415 genes were included. On average, there were 3.85 categories per gene, while each GO category contained an average of 10.2 genes. There were 16,997 GO category labels applied in total, covering $69.3 \%$ of the genome. On the other hand, 3,888 genes were labeled with an InterPro signature, averaging 3.09 labels per gene. There were 11,997 InterPro labels applied in total, covering $61.0 \%$ of the genome. Finally, the metabolic pathways of Plasmodium were extracted from the KEGG database, such that in total 182 metabolic pathways were detailed, averaging 36.7 genes, with pathways ranging from two genes to 342 genes. 2,938 genes were part of at least one pathway, averaging 2.3 pathways per gene. There were 6,671 metabolic pathway assignments applied in total, covering $46.1 \%$ of the genome.

Overall, 7,682 total labels were applied, assigning 5,184 genes to 6,427 gene sets. This accounts for $81.4 \%$ of the genome, each gene being a member on average of 9.2 sets, with the most labeled gene being represented in 45 sets. The portion of the genome that was not represented in these sets included genes having no ascribed functional annotation. Over- or under-representation of a group of functional categories in comparison to all other categories was determined using hypergeometric distribution. Any comparison with a P-value $<0.01$ was retained.

Hierarchical clustering was performed using PCC values obtained from all pairwise comparisons across all 11 transcriptome data sets using ratio of $\log _{2}$-transformed and normalized gene expression values.

\section{Quantitative real-time PCR and RT-PCR}

Real-time PCR was performed in a DNA Engine Opticon 2 detector (Bio-Rad, Hercules, CA, USA) to assess transcript levels and gene copy numbers using the iQ SYBR Green Supermix (Bio-Rad). Parallel amplification reactions were carried out in 96-well plates in $25 \mu \mathrm{L}$ volume containing 0.3 to $0.5 \mu \mathrm{M}$ of each forward and reverse primer, with all samples run in triplicates. Measurements were performed on three independent occasions. Primer sequences and real-time PCR conditions are listed in Additional file 27: Table S18.

For quantitative RT-PCR, RNA was treated with DNase I (Ambion) to remove possible traces of contaminating genomic DNA. cDNA was prepared by reverse transcription from $5 \mu \mathrm{g}$ of total RNA using a mix of oligodT (Invitrogen) and random hexamers (Invitrogen). To assess transcript levels, cDNA templates were diluted 60- to 80-fold. Relative quantification of transcripts was performed using the standard curve method, using 10fold dilution series of genomic DNA harvested from the parental strains 7G8 or FCB. cyp87 (PF3D7_0510200, previously PFE0505w, Forward 5' AAACGGGAGATC CTTCAGGT, Reverse $5^{\prime}$ AAGGACATGGGACAGTG GTT) was used as the reference control gene.

To assess gene copy numbers within $p f m d r 1$ amplicon, $50 \mathrm{ng}$ of template genomic DNA was used to measure each gene copy number relative to the single-copy cyp 87 or $\beta$-tubulin (PF3D7_1008700, previously PF10_0084) genes. Assays were normalized using standard curves that were generated by 10 -fold dilution series of genomic DNA from 3D7, known to have a single copy of pfmdr1 amplicon. Copy number results were rounded to the nearest integer. 


\section{Availability of supporting data}

The data sets supporting the results of this article are available in the GEO (Gene Expression Omnibus) functional genomics data repository at the NCBI, under the accession number GSE75807 (http://www.ncbi.nlm.nih.gov/geo/query/acc.cgi?acc=GSE75807).

\section{Additional files}

Additional file 1: Figure S1. Schema summarizing the parental and transgenic parasite lines used in this study. (A) The FCB data set comprises FCB, FCB ${ }^{\text {pfmdr } 13^{3^{\prime} K D}}$ and 106/1 (not pictured). FCB ${ }^{\text {pfmdr } 13^{3^{\prime} K D}}$ was derived from the multidrug-resistant strain FCB by genetically disrupting one of the two copies of the pfmdr 1 gene, as reported in [26]. pBS: pBluescript plasmid backbone. $\Delta m d r$ : pfmdr 1 fragment used to disrupt the coding sequence in one of the two endogenous tandem pfmdr 1 loci in FCB, resulting in FCB ${ }^{\text {pfmdr } 1-3^{\prime} K D}$ (knock-down). (B) The 7G8 data set comprises the CQ-resistant parental strain 7G8 (PfCRT haplotype T76), from which two transgenic lines were derived by allelic exchange as previously described [20], namely the control 7G8 $8^{\text {pfrt_CTL }}$ (moderately CQ-resistant, PfCRT haplotype T76) and the back-mutant $7 \mathrm{G}^{\text {pfort_ }}{ }^{\text {T76K }}$ that is now fully CQ-sensitive (PfCRT haplotype K76). (PDF 347 kb)

Additional file 2: Table S1. Validation of the data point imputation. Using the publically available transcriptome datasets for 3D7, Dd2 and HB3 from (Llinas et al. NAR 2007), we validated whether the density of the data in our time series (of eight points, at a 6-hour resolution) was sufficient to impute data to a $7.5 \mathrm{~min}$ resolution. For each strain, we selected eight time points evenly spread (6-hrs apart) from which we generated an imputed curve of 384 points. Using Pearson correlation values for gene expression, we then compared each actual hourly time point to the imputed curve to identify the closest matching time point The imputed percentage column shows where the match occurred in the time series. Actual time points from the original time courses match imputed time points that are fairly evenly spread along the imputation-derived curve. (XLSX $37 \mathrm{~kb}$ )

Additional file 3: Figure S2. Heatmaps showing alignment of each profiled transcriptome to that of 3D7. FFT was used to impute expression levels from the 8 sampled time points across a data set of 384 time points spanning the 48-hour IDC. This allowed expression level to be extrapolated with close temporal resolution (7.5 min) across the IDC. Dynamic time warping was used to align all 384 points to those of the published 3D7 time series [48, 70], using the PCC score computed between each pair of points. (PDF $2732 \mathrm{~kb}$ )

Additional file 4: Figure S3. Example of a chromosome map showing differential gene expression in the telomeric regions of chromosome 11 between FCB and both FCB ${ }^{\text {fmdri }}{ }^{3^{\prime} K D}$ and 106/1. Expression fold changes for both pairwise comparisons were plotted as a function of the chromosomal coordinates of each gene. Each circle represents a distinct gene. The dashed line delineates the threshold value of a 1.5 fold shift that was interpreted as evidence of transcriptional differences. Note that the (sub)telomeric regions of chromosome 11 are enriched in transcriptionally variable multigene families, notably var and phist genes. (PDF 129 kb)

Additional file 5: Table S2. List of genes within the pfmdr1-containing amplicon with associated fold change between FCB and both FCB ${ }^{\text {pfmdr }{ }_{3} 3^{\text {i }}}$ $K D$ and 106/1, as measured by microarrays. (XLSX $11 \mathrm{~kb}$ )

Additional file 6: Figure S4. Genomic map of the pfmdr1-containing amplicon region on chromosome 5 of the FCB strain. Genes listed in Additional file 2: Table S1 are indicated and pfdmr1 (PF3D7_052300) is boxed in red. (PDF $90 \mathrm{~kb}$ )

Additional file 7: Table S3. Results of quantitative real time PCR to estimate gene copy numbers associated with gene expression variations between FCB and both FCB_3'KD and 106/1 in the pfmdr1-containing amplicon region on chromosome 5 . These data suggest dosage compensation for the gene Pf3D7_0521300. (XLSX $57 \mathrm{~kb})$
Additional file 8: Table S4. Values for gene expression fold differences between strains in the 7G8 data set for genes on chromosome 2. The boxed area corresponds to the region on chromosome 2 containing genes with a significant fold change (values <0.6). (XLSX $13 \mathrm{~kb}$ )

Additional file 9: Figure S5. Venn diagrams depicting the number of genes that were found differentially expressed between FCB and (A) FCB ${ }^{\text {pfmdrl } 3_{3} K D}$ or (B) $7 G 8^{\text {pfcrt_CTL }}$ and $7 G 8^{\text {pfcrt_T76K }}$ during one or more developmental stages. Fold changes greater than 1.5 and normalized fold changes two standard deviations above the median value were used for the analyses. (PDF $82 \mathrm{~kb}$ )

Additional file 10: Table S5. Assessment of a possible time shift in gene expression between parasite lines. We examined the data to assess whether some genes displayed a shifted timing of expression between parasite strains. By comparing peaks of expression for each gene in each pairwise comparison, we determined the number of genes whose peak of expression was shifted of a determined number of hours. 0 corresponds to a shift of less than $1 \mathrm{~h}$, one corresponds to a shift of at least $1 \mathrm{~h}$ but less than two hours, etc. Therefore, two genes cannot be more than $24 \mathrm{~h}$ apart of each other in either direction. We observed that more than $70 \%$ of all genes peaked within the same 8-hour window in the pfmdr 1 dataset (Table S5A) while about $80 \%$ of the genes peaked within the same 5-hour window in the pfort dataset (Table S5B). (XLSX 44 kb)

Additional file 11: Table S6 Genes (A-D) and gene sets (E-H) differentially expressed in the pfmdr 1 data set. For each pairwise comparison, we listed genes with significant differential expression, i.e. with a fold change greater than 1.5 and a normalized fold change two standard deviations above the median values and genes with a fold change lower than 0.6 and a normalized fold change three standard deviations below the median value, which generally represented the quasi-totality of genes with a fold change below 0.6. Normalization was performed using the average fold change computed across a background pool of 11 transcriptome data sets as a scaling factor. This normalization allowed us to identify and filter out most of the hypervariable genes (e.g. var, rifin, stevor, phist, and hyp). Antigenically variant genes not detected by automatic filtering were manually removed. We listed the gene sets with significant differential expression (based on the mean rank of enrichment score $>0.9$ (significantly enriched) or $<0.1$ (significantly impoverished)) that are found in both FCB vs. FCB ${ }^{\text {pmandr } 13^{\prime} K D}$ and FCB vs. 106/1 pairwise comparisons. (XLSX 65 kb)

Additional file 12: Table S7. List of genes with significant differential expression between FCB and FCB ${ }^{\text {pmarl }} 3^{3} K D$ in a stage-specific manner. Genes with significantly higher expression displayed absolute fold change values $>1.5$ and normalized fold changes values two standard deviations above the median normalized fold change value. Fold change for each gene at the designated stage is indicated. No gene appeared to be significantly differently expressed between the two strains during both ring and schizont stages. Genes from antigenic variant families have been removed. (XLSX $71 \mathrm{~kb})$

Additional file 13: Table S8. List of genes with significant differential expression between FCB and 106/1 in a stage-specific manner. Genes with significantly higher expression displayed absolute fold changes $>1.5$ and normalized fold changes values two standard deviations above the median normalized fold change value. Genes with significantly lower expression had absolute fold change values lower than 0.6 and a normalized fold change three standard deviations below the median value. Fold change for each gene at the designated stage is indicated. (XLSX 66 kb)

Additional file 14: Figure S6. Gene set enrichment analysis for the

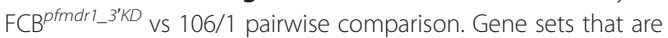
significantly $(\mathbf{A})$ enriched $(\mathbf{A})$ or $(\mathbf{B})$ depleted in $\mathrm{FCB}^{\text {ffmdr1_ } 3^{\prime} K D}$ in comparison with 106/1. We also performed an analysis of the overlap for (C) enriched and (D) depleted gene sets between FCB ${ }^{\text {pfmdr1 }{ }^{3} 3^{\prime} K D}$ vs 106/1 and FCB vs 106/1 comparisons. (PDF $162 \mathrm{~kb}$ )

Additional file 15: Table S9. Genes (A-F, I-K) and gene sets (G-H, L-M) differentially expressed in the pfcrt data set. For each pairwise comparison, $7 \mathrm{G} 8$ vs $7 \mathrm{G} 8^{\text {pfCrt_CTL }}, 7 \mathrm{G} 8$ vs $7 \mathrm{G} 8^{\text {pfCrt_T76K }}$, and $7 \mathrm{GG} 8^{\text {pfcrt_CTL }}$ vs 
7G8 $8^{\text {pfort_T76K }}$, we listed genes with significant differential expression, i.e. with a fold change greater than 1.5 and a normalized fold change two standard deviations above the median values. We listed the gene sets significantly enriched and depleted in 7G8 in comparison with both $7 \mathrm{G}^{\text {pfort_CTL }}$ and $7 \mathrm{G} 8^{\text {pfCrt_T76K }}$, and in $7 \mathrm{G} 8$ and $7 \mathrm{G}^{\text {pfrrt_CTL }}$ in comparison to $7 \mathrm{G}^{\text {pfert_T76K }}$. (XLSX $\left.48 \mathrm{~kb}\right)$

Additional file 16: Table S10. List of genes with significant differential

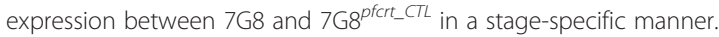
Genes with significantly higher expression displayed absolute fold changes $>1.5$ and normalized fold changes values two standard deviations above the median normalized fold change value. Genes with significantly lower expression had absolute fold change values lower than 0.6 and a normalized fold change three standard deviations below the median value. Fold change for each gene at the designated stage is indicated. (XLSX $55 \mathrm{~kb}$ )

Additional file 17: Table S11. Genes with significant differential expression between $7 \mathrm{G} 8$ and $7 \mathrm{G} 8^{\text {pfcrt_T76K }}$ in a stage-specific manner. Genes with significantly higher expression displayed absolute fold changes $>1.5$ and normalized fold changes values two standard deviations above the median normalized fold change value. Genes with significantly lower expression had absolute fold change values lower than 0.6 and a normalized fold change three standard deviations below the median value. Fold change for each gene at the designated stage is indicated. (XLSX $59 \mathrm{~kb}$ )

Additional file 18: Table S12. Genes with significant differential expression between $7 \mathrm{G} 8^{\text {pfcrt_CTL }}$ and $7 \mathrm{G} 8^{\text {pfcrt_T76K }}$ in a stage-specific manner. Genes with significantly higher expression displayed absolute fold changes $>1.5$ and normalized fold changes values two standard deviations above the median normalized fold change value. Genes with significantly lower expression had absolute fold change values lower than 0.6 and a normalized fold change three standard deviations below the median value. Fold change for each gene at the designated stage is indicated. (XLSX $58 \mathrm{~kb})$

Additional file 19: Figure S7. Gene sets with significant enrichment (orange) or depletion (blue) in 7G8 in comparison to $7 \mathrm{G} 8^{\text {pfort_CTL for }}$ three windows of the parasite IDC corresponding to rings $(R, 12-16 \mathrm{~h}$ post-invasion), trophozoites (T, 24-28 h post-invasion) and schizonts (S, 32-36 h post-invasion). (PDF $110 \mathrm{~kb}$ )

Additional file 20: Table S13. Hierarchical clustering, which was performed using Pearson correlation coefficients between $\sim 2600$ gene pairs across 55 pairwise comparisons of 11 parasite transcriptome data sets, including FCB and 7G8 data sets. Cluster 1 corresponding to gene pairs with high Pearson correlation (PCC $>0.7$ ), containing pfmdr1 and pfcrt as well as a transcription factor of the ApiAP2 family PF3D7_0516800. (XLSX 42 kb)

Additional file 21: Table S14. Cluster 2 from the Hierarchical clustering, corresponds to another group of gene pairs with high Pearson correlation (PCC >0.7) and includes pfmdr1 and pfcrt. (XLSX $46 \mathrm{~kb}$ )

Additional file 22: Figure S8. Cluster heatmap of gene expression data for pfcrt/pfmdr1 and all other genes. The hierarchical clustering was generated using PCC values calculated using $\log _{2}$-transformed and normalized expression values of 2,600 genes across 110 pairwise comparisons of 11 parasite transcriptome data sets, corresponding to all possible combinations in both directions (i.e. A vs. B and B vs. A). Squares indicate areas containing genes that are all strongly correlated or strongly anti-correlated with the expression of both pfcrt and pfmdr1. (PDF $499 \mathrm{~kb}$ )

Additional file 23: Table S15. Gene expression correlation between P. falciparum genes and either pfcrt or pfmdr1. Pearson correlation coefficients were calculated between pfcrt/pfmdr1 expression values and those of other 2,600 P. falciparum genes across 110 pairwise comparisons. These correspond to bi-directional comparisons between 11 parasite transcriptome data sets, including FCB and 7G8 data sets. (A) List of genes whose expression is highly correlated (PCC $>0.7$ ) to that of pfcrt. (B) List of genes whose expression is highly anti-correlated (PCC $<-0.7)$ to that of pfcrt. (C) List of genes whose expression is highly correlated (PCC >0.7) to that of pfmdr1. (D) List of genes whose expression is highly anti-correlated (PCC $<-0.7)$ to that of pfmdr1. Corresponding Spearman correlations are also indicated. (XLSX $87 \mathrm{~kb}$ )

Additional file 24: Table S16. Identification of genes whose expression is strongly correlated to that of both pfcrt and pfmr1. PCC were estimated from 110 pairwise comparisons of 11 transcriptome data sets. (A) List of genes organized by functional categories whose expression is highly correlated (PCC >0.7) to that of both pfcrt and pfmdr1. *Indicates genes with confidence, as found when using PCC computed from 55 unidirectional pairwise comparisons of the 11 transcriptome data sets. (B) List of genes organized by functional categories whose expression is highly anti-correlated (PCC $<-0.7)$ to that of both pfcrt and pfmdr1. *Indicates genes with confidence, as found when using PCC computed from 55 unidirectional pairwise comparisons of the 11 transcriptome data sets. (XLSX $42 \mathrm{~kb})$

Additional File 25: Zipped file folder containing code and pseudocode for HATS analysis. These files can be downloaded from http://www.fidock.org/data/fidock_source_code.zip. (DOCX 32 kb)

Additional File 26: Table S17. Table summarizing all the gene sets, extracted from various databases or built by our group, that were used in this study. (XLSX $688 \mathrm{~kb}$ )

Additional file 27: Table S18. List of the primers used for the GPCR and QRT-PCR reactions. (XLSX $31 \mathrm{~kb}$ )

\section{Abbreviations}

AUC: area under the curve; ACTs: artemisinin-based combination therapies; CQ: chloroquine; IDC: intra-erythrocytic developmental cycle; FDR: false discovery rate; FFT: Fast Fourier Transform; GO: gene ontology; GSEA: Gene Set Enrichment Analysis; HATS: Hypergeometric Analysis of Time Series; OPLS-DA: Orthogonal Partial Least Square-Discriminant Analysis; PCA: principal component analysis; PCC: Pearson correlation coefficient; PfCRT: Plasmodium falciparum Chloroquine Resistance Transporter; PfMDR1: Plasmodium falciparum Multidrug Resistance 1; SD: standard deviation.

\section{Competing interests}

The authors declare that they have no competing interests.

\section{Authors' contributions}

SHA, ML and DAF designed the experiments. SHA performed the experiments. EK assisted with the DNA microarray hybridizations. SHA, DS, ML and DAF analyzed the data. SHA, DS, and DAF wrote the manuscript. All authors read and approved the final manuscript

\section{Acknowledgements}

Funding for this work was provided in part by the NIH (R01 Al50234 and Al109023 to DAF, the NIH Director's New Innovators award 1DP2OD001315 to ML, and support for ML from the Center for Quantitative Biology P50 GM071508), as well as the Burroughs Wellcome Fund (New Investigators in Pathogenesis of Infectious Diseases) to DAF and ML. We thanks Michael Muhle for valuable discussions and help with the Orthogonal Partial Least Square-Discriminant Analysis.

\section{Author details}

'Department of Microbiology and Immunology, Columbia University Medical Center, New York, NY 10032, USA. ${ }^{2}$ Department of Molecular Biology \& Lewis-Sigler Institute for Integrative Genomics, Princeton University, Princeton, NJ 08544, USA. ${ }^{3}$ Department of Biochemistry and Molecular Biology, Department of Chemistry, Center for Malaria Research and Center for Infectious Diseases Dynamics, Pennsylvania State University, University Park, PA 16802, USA. ${ }^{4}$ Division of Infectious Diseases, Department of Medicine, Columbia University Medical Center, New York, NY 10032, USA. ${ }^{5}$ Present addresses: Wellcome Trust Sanger Institute, Hinxton, UK. ${ }^{6}$ Present addresses: Google Inc., New York, NY 10011, USA. ${ }^{7}$ Present addresses: Pulmonary Center, Boston University School of Medicine, Boston, MA 02118, USA.

Received: 7 July 2015 Accepted: 15 December 2015 Published online: 21 December 2015 


\section{References}

1. World Malaria Report 2015 - full report. http://www.who.int/malaria/ publications/world-malaria-report-2015/en/ 2014:1-242.

2. Alonso PL, Brown G, Arevalo-Herrera M, Binka F, Chitnis C, Collins F, et al. A research agenda to underpin malaria eradication. PLoS Med. 2011;8:e1000406.

3. White NJ, Pukrittayakamee S, Hien TT, Faiz MA, Mokuolu OA, Dondorp AM Malaria. Lancet. 2014;383:723-35.

4. Wellems TE, Plowe CV. Chloroquine-resistant malaria. J Infect Dis. 2001;184:770-6.

5. Naidoo I, Roper C. Mapping 'partially resistant', 'fully resistant', and 'super resistant' malaria. Trends Parasitol. 2013;29:505-15.

6. Dondorp AM, Nosten F, Yi P, Das D, Phyo AP, Tarning J, et al. Artemisinin resistance in Plasmodium falciparum malaria. N Engl J Med. 2009;361:455-67.

7. Phyo AP, Nkhoma S, Stepniewska K, Ashley EA, Nair S, McGready R, et al. Emergence of artemisinin-resistant malaria on the western border of Thailand: a longitudinal study. Lancet. 2012;379:1960-6.

8. Ashley EA, Dhorda M, Fairhurst RM, Amaratunga C, Lim P, Suon S, et al. Spread of artemisinin resistance in Plasmodium falciparum malaria. N Engl J Med. 2014;371:411-23.

9. Fidock DA, Nomura T, Talley AK, Cooper RA, Dzekunov SM, Ferdig MT, et al. Mutations in the $P$. falciparum digestive vacuole transmembrane protein PfCRT and evidence for their role in chloroquine resistance. Mol Cell. 2000;6:861-71.

10. Wilson CM, Serrano AE, Wasley A, Bogenschutz MP, Shankar AH, Wirth DF. Amplification of a gene related to mammalian mdr genes in drug-resistant Plasmodium falciparum. Science. 1989:244:1184-6.

11. Cowman AF, Galatis D, Thompson JK. Selection for mefloquine resistance in Plasmodium falciparum is linked to amplification of the pfmdrl gene and cross-resistance to halofantrine and quinine. Proc Natl Acad Sci U S A. 1994:91:1143-7.

12. Waller KL, Muhle RA, Ursos LM, Horrocks P, Verdier-Pinard D, Sidhu ABS, et al. Chloroquine resistance modulated in vitro by expression levels of the Plasmodium falciparum chloroquine resistance transporter. J Biol Chem. 2003;278:33593-601

13. Sidhu ABS, Valderramos SG, Fidock DA. pfmdr1 mutations contribute to quinine resistance and enhance mefloquine and artemisinin sensitivity in Plasmodium falciparum. Mol Microbiol. 2005:57:913-26.

14. Djimdé A, Doumbo OK, Cortese JF, Kayentao K, Doumbo S, Diourté Y, et al. A molecular marker for chloroquine-resistant falciparum malaria. N Engl J Med. 2001;344:257-63.

15. Sidhu ABS, Verdier-Pinard D, Fidock DA. Chloroquine resistance in Plasmodium falciparum malaria parasites conferred by pfcrt mutations. Science. 2002;298:210-13.

16. Picot $S$, Olliaro P, de Monbrison F, Bienvenu AL, Price RN, Ringwald P. A systematic review and meta-analysis of evidence for correlation between molecular markers of parasite resistance and treatment outcome in falciparum malaria. Malar J. 2009:8:89.

17. Baro NK, Callaghan PS, Roepe PD. Function of resistance conferring Plasmodium falciparum chloroquine resistance transporter isoforms. Biochemistry. 2013:52:4242-9.

18. Paguio MF, Cabrera M, Roepe PD. Chloroquine transport in Plasmodium falciparum. 2. Analysis of PfCRT-mediated drug transport using proteoliposomes and a fluorescent chloroquine probe. Biochemistry. 2009;48:9482-91.

19. Martin RE, Marchetti RV, Cowan Al, Howitt SM, Broer S, Kirk K. Chloroquine transport via the malaria parasite's chloroquine resistance transporter. Science. 2009:325:1680-2.

20. Lakshmanan V, Bray PG, Verdier-Pinard D, Johnson DJ, Horrocks P, Muhle RA, et al. A critical role for PfCRT K76T in Plasmodium falciparum verapamilreversible chloroquine resistance. EMBO J. 2005;24:2294-305.

21. Sisowath C, Petersen I, Veiga MI, Martensson A, Premji Z, Bjorkman A, et al. In vivo selection of Plasmodium falciparum parasites carrying the chloroquine-susceptible pfcrt K76 allele after treatment with artemetherlumefantrine in Africa. J Infect Dis. 2009;199:750-7.

22. Sa JM, Twu O, Hayton K, Reyes S, Fay MP, Ringwald P, et al. Geographic patterns of Plasmodium falciparum drug resistance distinguished by differential responses to amodiaquine and chloroquine. Proc Natl Acad Sci U S A. 2009;106:18883-9.

23. Reed MB, Saliba KJ, Caruana SR, Kirk K, Cowman AF. Pgh1 modulates sensitivity and resistance to multiple antimalarials in Plasmodium falciparum. Nature. 2000;403:906-9.

24. Conrad MD, LeClair N, Arinaitwe E, Wanzira H, Kakuru A, Bigira V, et al. Comparative impacts over 5 years of artemisinin-based combination therapies on Plasmodium falciparum polymorphisms that modulate drug sensitivity in Ugandan children. J Infect Dis. 2014;210:344-53.

25. Price RN, Uhlemann AC, van Vugt M, Brockman A, Hutagalung R, Nair S, et al. Molecular and pharmacological determinants of the therapeutic response to artemether-lumefantrine in multidrug-resistant Plasmodium falciparum malaria. Clin Infect Dis. 2006:42:1570-7.

26. Sidhu ABS, Uhlemann A-C, Valderramos SG, Valderramos J-C, Krishna S, Fidock DA. Decreasing pfmdr1 copy number in Plasmodium falciparum malaria heightens susceptibility to mefloquine, lumefantrine, halofantrine, quinine, and artemisinin. J Infect Dis. 2006;194:528-35.

27. Rohrbach P, Sanchez CP, Hayton K, Friedrich O, Patel J, Sidhu ABS, et al. Genetic linkage of pfmdr1 with food vacuolar solute import in Plasmodium falciparum. EMBO J. 2006:25:3000-11.

28. Hayward R, Saliba KJ, Kirk K. pfmdr1 mutations associated with chloroquine resistance incur a fitness cost in Plasmodium falciparum. Mol Microbiol. 2005:55:1285-95.

29. Froberg G, Ferreira PE, Martensson A, Ali A, Björkman A, Gil JP. Assessing the cost-benefit effect of a Plasmodium falciparum drug resistance mutation on parasite growth in vitro. Antimicrob Agents Chemother. 2013:57:887-92.

30. Rosenthal PJ. The interplay between drug resistance and fitness in malaria parasites. Mol Microbiol. 2013:89:1025-38.

31. Petersen I, Gabryszewski SJ, Johnston GL, Dhingra SK, Ecker A, Lewis RE, et al. Balancing drug resistance and growth rates via compensatory mutations in the Plasmodium falciparum chloroquine resistance transporter. Mol Microbiol. 2015;97:381-95.

32. Kublin JG, Cortese JF, Njunju EM, Mukadam RAG, Wirima JJ, Kazembe PN, et al. Reemergence of chloroquine-sensitive Plasmodium falciparum malaria after cessation of chloroquine use in Malawi. J Infect Dis. 2003;187:1870-5.

33. Laufer MK, Thesing PC, Eddington ND, Masonga R, Dzinjalamala FK, Takala SL, et al. Return of chloroquine antimalarial efficacy in Malawi. N Engl J Med. 2006;355:1959-66

34. Nair S, Nash D, Sudimack D, Jaidee A, Barends M, Uhlemann A-C, et al. Recurrent gene amplification and soft selective sweeps during evolution of multidrug resistance in malaria parasites. Mol Biol Evol. 2007;24:562-73.

35. Ord R, Alexander N, Dunyo S, Hallett R, Jawara M, Targett G, et al. Seasonal carriage of pfcrt and pfmdr 1 alleles in Gambian Plasmodium falciparum imply reduced fitness of chloroquine-resistant parasites. J Infect Dis. 2007;196:1613-9.

36. Laufer MK, Takala-Harrison S, Dzinjalamala FK, Stine OC, Taylor TE, Plowe CV. Return of chloroquine-susceptible falciparum malaria in Malawi was a reexpansion of diverse susceptible parasites. J Infect Dis. 2010;202:801-8.

37. Sherman DR, Mdluli K, Hickey MJ, Arain TM, Morris SL, Barry CE, et al. Compensatory ahpC gene expression in isoniazid-resistant Mycobacterium tuberculosis. Science. 1996;272:1641-3.

38. Handel A, Regoes RR, Antia R. The role of compensatory mutations in the emergence of drug resistance. PLoS Comput Biol. 2006;2:e137.

39. Nair S, Miller B, Barends M, Jaidee A, Patel J, Mayxay M, et al. Adaptive copy number evolution in malaria parasites. PLoS Genet. 2008:4:e1000243.

40. Brown KM, Costanzo MS, Xu W, Roy S, Lozovsky ER, Hartl DL. Compensatory mutations restore fitness during the evolution of dihydrofolate reductase. Mol Biol Evol. 2010;27:2682-90.

41. Kumpornsin K, Modchang C, Heinberg A, Ekland EH, Jirawatcharadech P, Chobson $\mathrm{P}$, et al. Origin of robustness in generating drug-resistant malaria parasites. Mol Biol Evol. 2014;31:1649-60.

42. McCollum AM, Mueller K, Villegas L, Udhayakumar V, Escalante AA. Common origin and fixation of Plasmodium falciparum dhfr and dhps mutations associated with sulfadoxine-pyrimethamine resistance in a low-transmission area in South America. Antimicrob Agents Chemother. 2007;51:2085-91.

43. Jiang H, Patel JJ, Yi M, Mu J, Ding J, Stephens R, et al. Genome-wide compensatory changes accompany drug- selected mutations in the Plasmodium falciparum crt gene. PLoS One. 2008;3:e2484.

44. Ganesan K, Ponmee N, Jiang L, Fowble JW, White J, Kamchonwongpaisan S, et al. A genetically hard-wired metabolic transcriptome in Plasmodium falciparum fails to mount protective responses to lethal antifolates. PLoS Pathog. 2008:4:e1000214.

45. Le Roch KG, Johnson JR, Ahiboh H, Chung D-WD, Prudhomme J, Plouffe D, et al. A systematic approach to understand the mechanism of action of the bisthiazolium compound T4 on the human malaria parasite, Plasmodium falciparum. BMC Genomics. 2008:9:513.

46. Bozdech Z, Llinás M, Pulliam BL, Wong ED, Zhu J, DeRisi JL. The transcriptome of the intraerythrocytic developmental cycle of Plasmodium falciparum. PLoS Biol. 2003;1:E5 
47. Le Roch KG, Zhou Y, Blair PL, Grainger M, Moch JK, Haynes JD, et al. Discovery of gene function by expression profiling of the malaria parasite life cycle. Science. 2003;301:1503-8.

48. Llinás $M$, Bozdech Z, Wong ED, Adai AT, DeRisi JL. Comparative whole genome transcriptome analysis of three Plasmodium falciparum strains. Nucleic Acids Res. 2006:34:1166-73.

49. Kidgell C, Volkman SK, Daily J, Borevitz JO, Plouffe D, Zhou Y, et al. A systematic map of genetic variation in Plasmodium falciparum. PLoS Pathog. 2006;2:e57.

50. Su X, Ferdig MT, Huang Y, Huynh CQ, Liu A, You J, et al. A genetic map and recombination parameters of the human malaria parasite Plasmodium falciparum. Science. 1999;286:1351-3.

51. Cooper RA, Ferdig MT, Su XZ, Ursos LM, Mu J, Nomura T, et al. Alternative mutations at position 76 of the vacuolar transmembrane protein PfCRT are associated with chloroquine resistance and unique stereospecific quinine and quinidine responses in Plasmodium falciparum. Mol Pharmacol. 2002;61:35-42.

52. Hu G, Llinás M, Li J, Preiser PR, Bozdech Z. Selection of long oligonucleotides for gene expression microarrays using weighted rank-sum strategy. BMC Bioinformatics. 2007:8:350.

53. Rovira-Graells N, Gupta AP, Planet E, Crowley VM, Mok S, de Pouplana Ribas $L_{\text {, }}$ et al. Transcriptional variation in the malaria parasite Plasmodium falciparum. Genome Res. 2012;22:925-38.

54. Balaji S, Babu MM, lyer LM, Aravind L. Discovery of the principal specific transcription factors of Apicomplexa and their implication for the evolution of the AP2-integrase DNA binding domains. Nucleic Acids Res. 2005;33:3994-4006.

55. Campbell TL, De Silva EK, Olszewski KL, Elemento O, Llinas M. Identification and genome-wide prediction of DNA binding specificities for the ApiAP2 family of regulators from the malaria parasite. PLoS Pathog. 2010;6:e1001165.

56. Subramanian A, Tamayo P, Mootha VK, Mukherjee S, Ebert BL, Gillette MA, et al. Gene set enrichment analysis: a knowledge-based approach for interpreting genome-wide expression profiles. Proc Natl Acad Sci U S A. 2005;102:15545-50.

57. Mok S, Ashley EA, Ferreira PE, Zhu L, Lin Z, Yeo T, et al. Drug resistance. Population transcriptomics of human malaria parasites reveals the mechanism of artemisinin resistance. Science. 2015;347:431-5.

58. Sullivan Jr DJ, Gluzman IY, Goldberg DE. Plasmodium hemozoin formation mediated by histidine-rich proteins. Science. 1996;271:219-22.

59. Siwo GH, Tan A, Button-Simons KA, Samarakoon U, Checkley LA, Pinapati RS, et al. Predicting functional and regulatory divergence of a drug resistance transporter gene in the human malaria parasite. BMC Genomics. 2015;16:115.

60. Gunasekera AM, Myrick A, Roch KL, Winzeler E, Wirth DF. Plasmodium falciparum: Genome wide perturbations in transcript profiles among mixed stage cultures after chloroquine treatment. Exp Parasitol. 2007;117:87-92.

61. Hu G, Cabrera A, Kono M, Mok S, Chaal BK, Haase S, et al. Transcriptional profiling of growth perturbations of the human malaria parasite Plasmodium falciparum. Nat Biotechnol. 2010;28:91-8.

62. Gonzales JM, Patel JJ, Ponmee N, Jiang L, Tan A, Maher SP, et al. Regulatory hotspots in the malaria parasite genome dictate transcriptional variation. PLoS Biol. 2008:6:e238.

63. Wiedemann N, Frazier AE, Pfanner N. The protein import machinery of mitochondria. J Biol Chem. 2004:279:14473-6.

64. Lewis IA, Wacker M, Olszewski KL, Cobbold SA, Baska KS, Tan A, et al. Metabolic QTL analysis links chloroquine resistance in Plasmodium falciparum to impaired hemoglobin catabolism. PLoS Genet. 2014;10:e1004085.

65. Volkman SK, Sabeti PC, DeCaprio D, Neafsey DE, Schaffner SF, Milner Jr DA, et al. A genome-wide map of diversity in Plasmodium falciparum. Nat Genet. 2007;39:113-9.

66. Folarin OA, Gbotosho GO, Sowunmi A, Olorunsogo OO, Oduola AMJ, Happi TC. Chloroquine resistant Plasmodium falciparum in Nigeria: relationship between pfcrt and pfmdr1 polymorphisms, in vitro resistance and treatment outcome. Open Trop Med J. 2008;1:74-82.

67. Price RN, Uhlemann A-C, Brockman A, McGready R, Ashley E, Phaipun L, et al. Mefloquine resistance in Plasmodium falciparum and increased pfmdr 1 gene copy number. Lancet. 2004;364:438-47.

68. Trager W, Jensen JB. Human malaria parasites in continuous culture. Science. 1976;193:673-5.

69. Kyes S, Pinches R, Newbold C. A simple RNA analysis method shows var and rif multigene family expression patterns in Plasmodium falciparum. Mol Biochem Parasitol. 2000;105:311-5.
70. Bozdech Z, Zhu J, Joachimiak M, Cohen F, Pulliam B, DeRisi J. Expression profiling of the schizont and trophozoite stages of Plasmodium falciparum with a long-oligonucleotide microarray. Genome Biol. 2003;4:R9.

71. Aach J, Church GM. Aligning gene expression time series with time warping algorithms. Bioinformatics. 2001;17:495-508.

72. Bylesjö M, Rantalainen M, Cloarec O, Nicholson JK, Holmes E, Trygg J. OPLS discriminant analysis: combining the strengths of PLS-DA and SIMCA classification. J Chem Theory Comput. 2007;20:341-51.

73. Lisewski AM, Quiros JP, Ng CL, Adikesavan AK, Miura K, Putluri N, et al. Supergenomic network compression and the discovery of EXP1 as a glutathione transferase inhibited by artesunate. Cell. 2014;158:916-28.

74. Mok S, Imwong M, Mackinnon MJ, Sim J, Ramadoss R, Yi P, et al. Artemisinin resistance in Plasmodium falciparum is associated with an altered temporal pattern of transcription. BMC Genomics. 2011;12:391.

75. Ashburner M, Ball CA, Blake JA, Botstein D, Butler H, Cherry JM, et al. Gene ontology: tool for the unification of biology. The Gene Ontology Consortium. Nat Genet. 2000;25:25-9.

76. Ginsburg H. Caveat emptor: limitations of the automated reconstruction of metabolic pathways in Plasmodium. Trends Parasitol. 2009;25:37-43.

77. Ginsburg H, Tilley L. Plasmodium falciparum metabolic pathways (MPMP) project upgraded with a database of subcellular locations of gene products. Trends Parasitol. 2011:27:285-6.

78. Kanehisa M. The KEGG resource for deciphering the genome. Nucleic Acids Res. 2004:32:277-80.

79. Mitchell A, Chang HY, Daugherty L, Fraser M, Hunter S, Lopez R, et al. The InterPro protein families database: the classification resource after 15 years. Nucleic Acids Res. 2015;43:D213-221.

\section{Submit your next manuscript to BioMed Central and we will help you at every step:}

- We accept pre-submission inquiries

- Our selector tool helps you to find the most relevant journal

- We provide round the clock customer support

- Convenient online submission

- Thorough peer review

- Inclusion in PubMed and all major indexing services

- Maximum visibility for your research

Submit your manuscript at www.biomedcentral.com/submit
) Biomed Central 